\title{
Model Simulations Unveil The Structure-Function- Dynamics Relationship of The Cerebellar Cortical Microcircuit
}

\section{Robin De Schepper}

University of Pavia

Alice Geminiani

University of Pavia

Stefano Masoli

University of Pavia https://orcid.org/0000-0003-0660-9952

Martina Francesca Rizza

University of Pavia

Alberto Antonietti

University of Pavia https://orcid.org/0000-0003-0388-6321

Claudia Casellato

University of Pavia

Egidio D'Angelo ( $\nabla$ dangelo@unipv.it )

University of Pavia https://orcid.org/0000-0002-6007-7187

\section{Article}

Keywords: computational modeling, structure-function relationship, cerebellum, neuronal,microcircuits, multi-compartmental neuron models

Posted Date: November 2nd, 2021

DOl: https://doi.org/10.21203/rs.3.rs-886369/v1

License: (a) (1) This work is licensed under a Creative Commons Attribution 4.0 International License. Read Full License

Additional Declarations: There is NO Competing Interest.

Version of Record: A version of this preprint was published at Communications Biology on November 14th, 2022. See the published version at https://doi.org/10.1038/s42003-022-04213-y. 
1 Model simulations unveil the structure-function-dynamics relationship of

2

3 Robin De Schepper ${ }^{1}$, Alice Geminiani ${ }^{1}$, Stefano Masoli ${ }^{1}$, Martina Francesca Rizza ${ }^{1}$, Alberto Antonietti ${ }^{1}$,

4 Claudia Casellato ${ }^{1, \#}$, Egidio D'Angelo ${ }^{1,2, \#}$

$5 \quad{ }^{1}$ Department of Brain and Behavioral Sciences, University of Pavia, Pavia, Italy.

$6 \quad{ }^{2}$ IRCCS Mondino Foundation, Brain Connectivity Center, Pavia, Italy.

$7 \quad$ \# Co-last and corresponding authors

8

9 Running title: Realistic cerebellar cortex model

Keywords: computational modeling, structure-function relationship, cerebellum, neuronal microcircuits, multi-compartmental neuron models

\section{Abstract}

The cerebellar network is renowned for its regular architecture that has inspired foundational computational theories. However, the relationship between circuit structure, function and dynamics remained elusive. To tackle the issue, we have developed an advanced computational modeling framework that allowed us to reconstruct and simulate the structure and function of the mouse cerebellar cortex using morphologically realistic multi-compartmental neuron models. The cerebellar connectome was generated through appropriate connection rules, unifying a collection of scattered experimental data into a coherent construct and providing a new model-based ground-truth about circuit organization. Naturalistic background and sensory-burst stimulation were then used for functional validation against recordings in vivo, monitoring the impact of cellular mechanisms on signal propagation and spatiotemporal processing. Our simulations show, for the first time, how mossy fibers entrain the local neuronal microcircuit boosting the formation of columns of activity travelling from the granular to the molecular layer providing a new resource for the investigation of cerebellar computation.

\section{Introduction}

The relationship between structure, function and dynamics in brain circuits is still poorly understood posing a formidable challenge to neuroscience ${ }^{1}$. The core of the issue is how to deal with the distribution and causality of neural processing across multiple spatio-temporal scales. While experimental measurements remain essential, they can now be supported and complemented by realistic computational models. In principle, such models could take into account multi-modal datasets representing morphology, connectivity and activity of different cell populations and make it possible to simulate the propagation of microscopic phenomena into large-scale network dynamics ${ }^{2-4}$. These models can incorporate a broad range of biological data becoming highly constrained and providing the 
best proxies of the corresponding natural circuits. Eventually, once properly configured and validated, these models can generate their own ground-truth by binding the many parameters, provided by independent measurements and intrinsically prone to experimental error, into a coherent construct, and can be used to test various functional hypotheses ${ }^{5}$ using specific simulations platforms, like NEURON ${ }^{6}$ and NEST ${ }^{7}$. There are several examples of advanced computational models that have been mostly developed to simulate activities in the cerebral cortex ${ }^{8-10}$. Here we have developed a specific simulator, the Brain Scaffold Builder (BSB), to cope with the organization of the cerebellar network.

The cerebellar cortical microcircuit has inspired foundational theories on brain functioning ${ }^{11}$ but still challenges realistic computational modeling ${ }^{12}$. Previous network models using ionic conductancebased neurons have been developed only for the granular layer ${ }^{13,14}$. The only model encompassing the granular and molecular layer altogether made use of single-point neurons with a simplified representation of membrane excitability ${ }^{15}$. Although those models showed a remarkable predictive power against specific target parameters, their main limitation was that connectivity was set independently from neuronal morphology ${ }^{13-15}$ preventing a direct link between microcircuit structure, function and dynamics. In the meanwhile, detailed computational models of the main cerebellar cortical neurons, which were based on morphological reconstructions embedding multiple membrane ionic channels and synaptic receptors, have been developed, tested and validated ${ }^{16-19}$. Thus, with the BSB, we have been able to generate the first computational model of the entire cerebellar cortical microcircuit including both the granular and molecular layer, in which multicompartmental neuron models were wired through a connectome defined by the anisotropy of dendritic and axonal processes through principled rules. The model allowed then to simulate network dynamics and validate it against naturalistic inputs ${ }^{20-22}$.

This work generates de facto a new model-based ground-truth for the cerebellar cortical microcircuit, predicting the weight that some connections should have to balance the internal activity. On the scale used here, there are two main emerging spatio-temporal dynamics. First, background mossy fiber bombardment induces coherent oscillations throughout the granular layer. Secondly, collimated mossy fibre bursts mimicking punctuate sensory stimulation generate dense clusters of granule cell activity that propagate vertically and invade the overlaying molecular layer. The emergence of these responses, which may represent the basis of local computation in the cerebellar microcircuit, anticipates properties revealed experimentally in vivo suggesting that the BSB cerebellar model will provide a valid resource for future cerebellar investigations.

\section{Results}




\subsection{The Brain Scaffold Builder (BSB)}

Cerebellar modelling using realistic morphologies poses specific problems, mostly related to the anisotropy and regular geometry of the network, that are not easily manageable with existing modeling tools ${ }^{8-10}$ so that we developed a new simulation platform, the BSB. The BSB allowed to easily solve construction problems like the precise orientation of neuron process in the 3D space, the connectivity of neurons through prescribed rules dictated by anatomo-physiological measurements and the choice of a variety of intersection rules depending on network geometry. The BSB operated through a sequence of independent steps: network configuration, reconstruction and simulation (Fig. 1a). The network volume was defined first along with cell types, then the BSB proceeded with cell placement and connectivity, reconstructing the microcircuit network (Fig. 1b, c). Finally, the BSB was interfaced with the NEURON simulator and network activity was simulated and the results visualized. Details on BSB operations are given in the Methods and Supplemental Material.

\subsection{Cerebellar network reconstruction}

The BSB was applied to the mouse cerebellar cortical network, which has a geometrically organized architecture that has been suggested to imply its computational properties ${ }^{11,12}$. The reconstruction and simulation of a network volume of $17.710^{-3} \mathrm{~mm}^{3}$ is reported, including the following cell and fiber types: mossy fiber $(\mathrm{mf})$, glomerulus ( $\mathrm{glom})$, granule cell $(\mathrm{GrC})$ with ascending axon $(\mathrm{a} a)$ and parallel fiber $(p f)$, Golgi cell $(G o C)$, Purkinje cell $(P C)$, and molecular layer interneurons $(M L I)$ comprising stellate cells $(S C)$ and basket cells $(B C)$.

\subsubsection{Neuron placement}

The network elements summed up to $29 \cdot 230$ neurons $(G r C, G o C, P C, S C, B C)$ plus $2 * 453$ other elements ( $m f$, glom), which were placed in the network volume according to anatomical data ${ }^{12,13,23}$ (Fig. 2a). The density values matched the targets given in the configuration file, the nearest neighbour and the pairwise distance distribution always exceeded cell diameter, and radial distribution function demonstrated the homogeneity of cell distribution without overlapping (Fig. S1).

\subsubsection{Neuron connectivity}

The network connections summed up to $1^{\circ} 500^{\circ} 000$ chemical synapses and $2 \cdot 100$ electrical synapses. The cerebellar connectome was modelled combining probabilistic and geometric rules that were chosen depending on available data and the nature of fiber (axon and dendrites) crossing (Fig. $2 \mathrm{~b}, \mathrm{c}, \mathrm{d}$; see Methods for details). This flexible management of connections rules is unique and fixes problems not easy to solve with cerebral cortex simulators, which deal with isotropic cellular organizations and adopt a limited number of intersection rules for all neurons and connections ${ }^{8-10}$. 
The well-known connectivity of $m f$ and glom was entirely accounted for by literature data. The BSB generated local anisotropic glom clusters extending $60 \mu \mathrm{m}$ along the $x$-axis and $20 \mu \mathrm{m}$ along the $z$-axis ${ }^{24}$, with $\sim 20$ gloms per $m f^{25}$. Imposing that each $G r C$ sends its 4 dendrites to gloms belonging to different $m f$ s within about $30 \mu \mathrm{m}$, the BSB yielded $49 \mathrm{GrCs}$ per glom on average ${ }^{26,27}$. Each of the 4 $\mathrm{GrC}$ dendrites, in addition to a single excitatory synapse on the terminal compartment, also hosted 1 inhibitory synapse on the preterminal compartment, mostly originating from different $G o C$ s (Fig. 2c) $28,29$.

The connectivity of $\mathrm{GoCs}$ was faced using either literature data ( $\mathrm{glom}-\mathrm{GoC}$ ) or adopting various intersection rules ( $a a-G o C, p f-G o C, G o C-G o C$ ). In fair agreement with literature, each $G o C$ received excitation from 56 different gloms and each glom collected basolateral dendrites from $\sim 2 \mathrm{GoCs}{ }^{30}$. There were $320 a a$ synapses on basolateral dendrites and $910 \mathrm{pf}$ synapses on apical dendrites per $\mathrm{GoC}$, all from different $\mathrm{GrCs}$ (Fig. 2b) ${ }^{31}$. Moreover, each $\mathrm{GoC}$ received inhibition from 16 other $\mathrm{GoCs}{ }^{32}$ on basolateral dendrites (subsequent functional calibration implied $~ 160$ synapses per pair, see below). Finally, there were $\sim 8 G o C$ s that formed gap junctions on other $G o C s$, with $\sim 3.5$ gap junctions per pair 33.

The connectivity of $P C \mathrm{~s}$ and $M L I \mathrm{~s}$ was recovered using suitable intersection rules ( $a a-P C, p f-$ $P C$, and all $M L I$ synapses). The BSB identified 1.500 $p f$ synapses per $P C$ (this figure was limited by the 200- $\mu$ m network size along $z$-axis but it would range up by 1 order of magnitude in an unbounded volume ${ }^{18,3418}$ ) and $197 a a$ synapses per $P C$ from 82 different $G r C s{ }^{35}$. There were $480 p f$ synapses per $S C$ and $740 p f$ synapses per $B C$, while $M L I$ reciprocal inhibition ${ }^{36}$ involved $14 S C$-SC and $14 B C$-BC connections with $\sim 100$ synapses per pair. The $S C$ axon, mainly extending on the coronal plane, innervated $\sim 2 P C \mathrm{~s}^{37}$ and each $P C$ received synapses from $\sim 5 S C$ s (Fig. S2). The $B C$ axon, mainly extending on the sagittal plane, innervated $\sim 14 P C$ s and each $P C$ received synapses from $\sim 20 B C$ s (akin with the figure of 3-50 baskets around the $P C$ soma and 7-10 PCs per $B C)^{37,38}$. These predictions of structural parameters were further assessed and tuned through functional simulations (see below).

\subsection{Cerebellar network simulations}

Network simulations were carried out using detailed neuronal and synaptic models written in NEURON for $G r C^{17}, G o C^{16}, P C,{ }^{18}, S C$ and $B C^{19}$. Local microcircuit responses to input patterns were tracked back to individual neurons and used to follow signal propagation with unprecedented resolution. All simulations were carried out in the presence of background noise to improve comparison with recordings in vivo. The emerging spatio-temporal dynamics provided functional model validation beyond constructive validity based on internal connectivity and single neuron responses (Movie S1). 


\subsubsection{Resting state activity of the cerebellar network}

A random input at low frequency ( $4 \mathrm{~Hz}$ Poisson) on all $m f \mathrm{~s}^{20}$ was used to simulate the cerebellar network in resting state in vivo. Since anatomical data about the connectivity of cerebellar neurons are incomplete, but their resting discharge frequency is known, we finetuned the number of connections per pair against target values of basal discharge. The turning point was to calibrate $G o C$-GoC inhibition, which influenced resting state activity of the entire network. Since the synaptic conductance $(\sim 3200$ $\mathrm{pS}$ ) and the number of interconnected GoCs (about 15) are known ${ }^{32}$, we tuned the number of $G o C$ $G o C$ synapses until basal discharge frequency was achieved. Eventually, the background frequency of all cerebellar neuron types fell in the ranges reported in vivo in anaesthetized rodents $(m f \mathrm{~s}: 4.2 \pm 2.6$ Hz; GrCs: $0.81 \pm 1.3 \mathrm{~Hz}$; GoCs: $19 \pm 15 \mathrm{~Hz}$; PCs: $31 \pm 1.6 \mathrm{~Hz}$; BCs: $11 \pm 5.1 \mathrm{~Hz}$; SCs: $9.4 \pm 12 \mathrm{~Hz})$ $\left[G r C \mathrm{~s}^{39}, G o C s,{ }^{16,40,41}, P C \mathrm{~s}^{42}, S C \mathrm{~s}\right.$ and $\left.B C \mathrm{~s}^{43-45}\right]$.

Background $m f$ activity is known to generate synchronous low-frequency oscillations in the granular layer ${ }^{46}$. Indeed, in the model, the FFT of $\mathrm{GoC}$ and $\mathrm{GrC}$ firing revealed a synchronous oscillatory behaviour in the theta band, with the first harmonic peaking at $9.7 \mathrm{~Hz}$. When GoC-GoC gap junctions were switched off, the regularity of the oscillation decreased and the first FFT harmonic moved out of theta band (Fig. 3a) ${ }^{47}$.

\subsubsection{Impulsive response of the cerebellar network}

Short stimulus bursts were delivered to a bundle of $4 \mathrm{mfs}$ connected to $\sim 80 \mathrm{gloms}$ to emulate whisker/facial sensory stimulation in vivo ${ }^{20,39}$. The burst propagated through the network, temporarily raising neuronal firing (Fig. 3b, Movie S1). The relationship between the number of spikes at afferent synapses and the response frequency to the $m f$ burst was robustly captured by multiple linear regression (Fig. 3c; Fig. S3a; Fig. S4).

\subsubsection{GrC responses}

Fundamental predictions on how $\mathrm{GrCs}$ respond to incoming bursts derive from current clamp recordings in situ ${ }^{48}$ and simulations ${ }^{17}$, which revealed the role of synaptic receptors and ionic channels. In BSB simulations, bursts on a collimated $m f$ bundle activated a dense cluster of $G r C s{ }^{15,21,49}$. The relationship between the number of input spikes (both at $\mathrm{GoC}-\mathrm{GrC}$ and $\mathrm{glom}-\mathrm{GrC}$ synapses) and $\mathrm{GrC}$ response frequency unveiled 4 groups of $\mathrm{GrCs}$ with a corresponding number of synaptically activated dendrites (Fig. 3c). The number of $G r C$ spikes, first spike latency and dendritic $\left[\mathrm{Ca}^{2+}\right]_{\text {in }}$ correlated with the number of active dendrites (NMI $=0.71,0.86,0.59$, respectively) (Fig. 4a,b).

When the inhibitory mechanisms (comprising transient and persistent inhibition) were switched off to simulate a pharmacological GABA-A receptor blockade, (i) $\mathrm{GrC}$ baseline frequency increased, (ii) a tail discharge appeared after the burst, (iii) responses including more spikes appeared, (iv) the first spike latency decreased, and (v) response variability decreased (Fig. 4a,b). The number of $\operatorname{GrC}$ spikes, first spike latency and dendritic $\left[\mathrm{Ca}^{2+}\right]_{\text {in }}$ still correlated with the number of active dendrites $(\mathrm{NMI}=$ 
$0.79,0.85,0.61$, respectively) (Fig. $4 \mathrm{~b}$ ). Interestingly, inhibition caused a reduction in the number of active $G r C$ s (i.e. those firing >= 1 spike in the $40 \mathrm{~ms}$ after the $m f$ burst onset were $3390 \pm 431$, and 8348 \pm 1724 with GABA-A off; $\mathrm{n}=10$ simulations; $\mathrm{p}<0.001$, unpaired $t$-test) but enriched the spike pattern, as predicted theoretically ${ }^{11,50}$.

Recordings in vivo disclosed precise integration of quanta and high-fidelity transmission in the granular layer ${ }^{20,51-54}$. In BSB simulations, $\mathrm{GrCs}$ receiving maximum excitation generated one action potential for each spike of the input burst, with short latency $(<2 \mathrm{~ms})$, and faithfully followed the input up to $250 \mathrm{~Hz}$ (Fig. 4a) (Movie S2).

\subsubsection{GoC responses}

Following punctuate sensory stimulation in vivo, GoCs have been reported to respond with short bursts of 2-3 spikes at up to $200-300 \mathrm{~Hz}^{55}$. In BSB simulations, GoCs immersed in the $\mathrm{GrC}$ active cluster generated a burst of 2-5 spikes with a maximum instantaneous frequency of $213 \pm 29 \mathrm{~Hz}$ (Fig. 4c). When GABA synapses and gap junctions between $G o C$ s were switched off, the response bursts showed up to 6 spikes, with a higher maximum instantaneous frequency $(308 \pm 16 \mathrm{~Hz})(\mathrm{n}=70$ GoCs; $\mathrm{p}<0.001$, paired $t$-test) (Fig. 4c). The burst was caused by synaptic excitation relayed by gloms and $G r C s$ (through both $a a$ s and $p f s$ ), which generated AMPA and NMDA currents in GoC dendrites (Movie S3). The "silent pause" appearing after the burst was caused both by an intrinsic phase-reset mechanism ${ }^{55-57}$ and by reciprocal inhibition between $G o C s$, demonstrating marked dendritic processing capabilities 16

\subsubsection{PC and MLI responses GrCs}

$P C \mathrm{~s}$ in vivo are known to respond to punctuate stimulation with burst-pause patterns ${ }^{22,58}$. In BSB simulations, $P C$ responses depended on cell position relative to the $m f$ active bundle (Fig. 5a). The $P C$ s placed vertically on top of the $G r C$ active cluster received the largest number of $a a$ and $p f$ synaptic inputs producing typical burst-pause patterns ${ }^{18}$. The burst coefficient was correlated with the number of synaptic inputs from $p f$ and $a a$ (multiple regression analysis: $\mathrm{R}^{2}=0.91$ ) (Fig. 5b). The pause coefficient was correlated with the burst coefficient (NMI=0.79) and with the number of spikes from MLIs (NMI=0.66) (Fig. 5b), reflecting the origin of the pause from both intrinsic after-hyperpolarizing mechanisms and $M L I$ inhibition ${ }^{59}$. Indeed, $M L I$ s are known to narrow the time window and reduce the intensity of $P C$ responses ${ }^{43}$. In BSB simulations, the $P C$ AMPA current arose soon after the spikes emitted by $G r C s$, while the PC GABA current was delayed by $2.6 \mathrm{~ms}$ (Fig. 5c). In summary, the disynaptic IPSCs produced by MLIs quickly counteracted the monosynaptic EPSCs produced by aas and $p f$ s, providing precise time control over $P C$ activation ${ }^{49,60}$.

$B C$ s in vivo are known to generate lateral inhibition reducing $P C$ discharge below baseline causing contrast enhancement ${ }^{43,50}$. In BSB simulations, this pattern emerged during stimulation of a $m f$ bundle (100 ms @ $50 \mathrm{~Hz}$ stimulation on 24 neighboring $m f \mathrm{~s}$ ). The $P C \mathrm{~s}$ placed in a band $150-200 \mu \mathrm{m}$ beside the active cluster along the $x$-axis were inhibited, bringing their frequency below baseline. When 
MLI-PC synapses were switched off, the effect disappeared revealing contrast enhancement due to lateral inhibition (Fig. 5d).

The response of MLIs in vivo is only partially known ${ }^{50}$. In BSB simulations, $S C$ s and $B C \mathrm{~s}$ intersected by active $p f \mathrm{~s}$ responded to input bursts and their activity remained higher than baseline for several hundreds of milliseconds, especially in $S C \mathrm{~s}^{19}$ (Fig. S3b).

\section{Discussion}

This work shows the first detailed model reconstruction and simulations of the cerebellar cortical network and predicts neuronal activities involved in the propagation of mossy fiber input signals from the granular to the PC and molecular layer. By means of the BSB model, we have combined heterogenous data using suitable placement and connectivity rules with accurate multi-compartmental neuron models. In the optimization process, the model extracted information from the interdependence of parameters, bound at high-level through ensuing network dynamics, allowing us to fill gaps in knowledge through constructive rules. In the validation process, the model demonstrated its compatibility with a wealth of experimental literature data collected over the last decades.

\subsubsection{A model-based ground-truth for the cerebellar cortical network}

The statistical and geometrical rules derived from anatomical and physiological works ${ }^{12,25}$ almost completely anticipated network connectivity at the cerebellar input stage. In the BSB model, each glom hosted $\sim 50$ excitatory and $\sim 50$ inhibitory synapses on as many $G r C$ dendrites, plus $\sim 2$ excitatory synapse on basolateral dendrites of as many GoCs, summing up to 102 synapses per glom, in agreement with the anatomical upper limit of $\sim 200^{28}$. Each one of the $4 \mathrm{GrC}$ dendrites received an excitatory and (in most cases) an inhibitory input from as many different $m f s$ and $G o C$ s, respectively 29,30. Each $G o C$ received $\sim 320 a a$ synapses on basolateral dendrites and $\sim 910 p f$ synapses on apical dendrites, according to the figure of $\sim 400$ and $\sim 1200^{31}$, and there were $\sim 3$ electrical synapses per $G o C$ $G o C$ pair ${ }^{47}$. Only the number of GoC-GoC GABAergic synapses, which amounted to a figure of 160 after functional tuning, lacked an experimental counterpart. In the molecular layer, under geometric and functional constraints, the BSB model placed limits to the debated numbers determining $P C$ and $M L I$ connectivity. The model predicted that $\sim 25 \%$ of aas contacted the distal dendrites of the overlaying $P C$ s ( $\left(7^{*} 133\right.$ out of $28^{*} 615 G r C s$ ), each $a a$ forming 2.4 synapses on average, supporting the important role predicted for the $a a^{60,61}$, while $p f$ s formed 1 synapse per $P C$ dendritic intersection. In summary, each $P C$ received $12 \%$ of the whole $G r C$ inputs from aas, matching the empirical estimate of $7-24 \%{ }^{62}$. The BSB generated $\sim 25 S C-P C$ and $B C$ - $P C$ synapses altogether, which compares well with the experimental estimate of $\sim 20^{63}$. Moreover, there were $\sim 17^{\circ} 600 \mathrm{pf}$-MLI-PC synapses $\left(\sim 2^{\circ} 600 \mathrm{pf}\right.$-SC- 
$P C$ and $\sim 15^{\circ} 000 p f-B C-P C$ synapses), compatible with the prediction that the $p f-M L I s-P C$ input is larger than the $p f-P C$ input on the same $P C^{64}$. In general, since all dendritic trees in the molecular layer are orthogonal to $p f s$, the BSB reconstruction ranked the number of synapses according to dendritic size $-P C(\sim 1 \cdot 500)>G o C(\sim 900)>B C(\sim 700)>S C(\sim 500)$ - a figure that would increase proportionately by scaling the model slab to include full-length $p f \mathrm{~s}{ }^{65}$.

Accurate single neuron models with realistic morphology proved also critical to carry out simulations allowing us to finetune the connectome. In particular, the number of inhibitory synapses per $G o C-G o C$ pair was increased in order to make them fire at $\sim 19 \mathrm{~Hz}\left[2-30 \mathrm{~Hz}\right.$ range $\left.{ }^{39,55}\right]$. Similarly, the number of inhibitory synapses per $S C-S C$ and per $B C-B C$ pairs was tuned in order to make them fire at $\sim 10 \mathrm{~Hz}\left[1-35 \mathrm{~Hz}\right.$ range $\left.{ }^{43,44}\right]$ and to bring $P C$ s into their resting state frequency range of $\sim 31 \mathrm{~Hz}$ $\left[36.4 \pm 11.5 \mathrm{~Hz}^{42,66}\right]$ in vivo.

Thus, a reconstruction of model connectivity purely based on geometrical rules was not sufficient and a careful tuning against functional data was needed. This two-pronged (structural and functional) approach ensured that all parameters were bound at high-level through the basal neuronal firing frequency at rest in vivo ${ }^{1}$. Eventually, the network connectome is in fair agreement with a wealth of disparate anatomical and functional determinations, suggesting that the emerging picture provides a new model-based ground-truth for the cerebellar cortical network.

\subsubsection{Cerebellar network model validation}

The functional validation of single neuron models was previously reported in specific studies ${ }^{16-19}$, so that these neurons could be directly plugged in and used to simulate spatio-temporal network dynamics in vivo. A first validation of the cerebellar network model implied responses to diffused background noise, which is reported to generate coherent large-scale oscillations ${ }^{46}$. The BSB model showed indeed that $\mathrm{GrCs}$ and $\mathrm{GoCs}$ were entrained into low-frequency coherent oscillations in resting state and, interestingly, this happened under gap junction control as shown experimentally ${ }^{47}$. A second functional validation was obtained by simulating responses to naturalistic $m f$ bursts, which rapidly propagated through the GrC-PC neuronal chain (Fig. 6) (Movie S1). GrCs responded in a dense cluster ${ }^{49}$ regulated by $G o C$ s and activated soon thereafter the overlaying $P C$ s and $M L I$ s. In the cluster, $45 \%$ of the GrCs fired at least one spike, in agreement with results reported previously ${ }^{12,49}$. Not unexpectedly, $S C$ s and $B C$ s effectively reduced activation of $P C$ s placed either along or beside the active $p f$ s, respectively, generating feedforward and lateral inhibition ${ }^{11,12}$. PCs showed the typical burstpause responses that are thought to correlate with cerebellar-dependent behaviors ${ }^{58}$. As a proof of model sensitivity, these response patterns were seriously altered by changing microscopic parameters, such as $m f-G r C$ neurotransmitter release probability ${ }^{17}$, whose effect propagated from the cerebellar input stage throughout the whole thickness of the cerebellar cortical network. 


\subsubsection{Limitations and future challenges}

The most significant problem in this kind of microcircuit models is to incorporate so many variables that they might be underconstrained. Here we have 5 cell types, 16 synaptic types and as many ranges for synaptic density. Almost all of them were carefully validated beforehand, except the BC model with its synapses and the density of reciprocal interneuron inhibitory synapses, which warrants specific investigation. Thus, although the parameterization of the cerebellar network model relies on one of the best-defined anatomical and physiological datasets in the brain ${ }^{12-14,67}$, it cannot be excluded that other parameter combinations might also be effective. Here we have enforced a connectivity principle largely based on proximity rules between neurites and tuned the connection algorithms to bring the connectivity within the anatomo-physiological range (see Methods). Alternative algorithms for automatic parameter tuning may also be used to predict the cerebellar cortical network connectome ${ }^{68}$ and compared to the present results. Finally, while we have used two most representative functional templates (background oscillations and response to sensory-burst stimulation in vivo), others could be envisaged.

Although it is validated on a small network scale (30 k neurons and $1.5 \mathrm{M}$ synapses), the model is about 1000 times smaller than the whole mouse cerebellum. This would not be a problem if the model would be a small-scale representation of the cerebellar cortex, but this is not the case given the anisotropy of cerebellar network architecture. The first issue is that signal propagation along the transverse plane would require longer modules. Here we have observed the formation of vertical columns ${ }^{60,62}$ but it would be important now to assess ${ }^{69,70}$ the beam hypothesis along with spatial signal filtering and plasticity ${ }^{11,50,16-19}$. Moreover, the cerebellar cortex is subdivided into microzones with different biochemical and functional properties, while the present model can just be taken as a good proxy of the $\mathrm{Z}+$ microzone ${ }^{71-73}$. Therefore, the model should be extended and diversified to explore effects on a larger scale.

Another issue is that, in the model, all neurons of the same type are identical one to another. However, there is morphological and functional variability among neurons of the same type. Moreover, there are known variants of granule cells, Golgi cells and Purkinje cells ${ }^{16-18}$. It would therefore be important to explore the impact of neuronal variability, which can bring about relevant computational effect ${ }^{74}$. The same also applies to synapses, which now have the same release probability and gain at homologous connections but are tuned by plasticity in real life ${ }^{16,21,22,34,64,67,72}$ and could therefore change network dynamics. The future introduction of plasticity, which now is present only in simplified models 75,76 and cerebellar subnetworks ${ }^{11-15}$, will allow to refine the effective functional organization of the connectome and test hypotheses on network functioning.

Finally, the operations of the cerebellar cortex are tightly bound to those of the deep cerebellar nuclei and of the inferior olive. However, to date the only available representations on the mesoscale are reconstructed using simplified single point neurons ${ }^{75,76}$ and a fine grain realistic representation is 
322 missing. Therefore, the model could be extended to the mesoscale to investigate how the cerebellar 323 cortex operates inside the olivo-cerebellar system.

\subsubsection{Conclusions}

In aggregate, the BSB model shows that the geometrical organization of neurites largely 328 determines cerebellar cortical connectivity and microcircuit dynamics, supporting the original intuition 329 of J.C. Eccles in the late 60 's ${ }^{11,50}$. A similar conclusion was recently reported for the cortical 330 microcolumn ${ }^{4}$. With appropriate extension, the model could allow to simulate cerebellar modules 331 including differentiated microzones and microcomplexes ${ }^{71-73}$ and more complex patterns of stimuli in 332 the sensorimotor and cognitive domain ${ }^{67}$. Given the "scaffold" design, new neurons and mechanisms 333 can be plugged-in to address ontogenesis, species differences (for example in humans) and pathology. 334 For example, the model may be used to predict the emerging dynamics caused by genetic or epigenetic 335 alterations in neuron (morphology and function) and synaptic properties, as it is supposed to happen in 336 ataxia, dystonia and autism ${ }^{77,78}$. The model may also be used to predict the impact of drugs acting on 337 ionic channels and synaptic receptors. In conclusion, the model can be regarded as a new resource for 338 investigating the cerebellar network. 


\subsection{The BSB modeling framework}

The BSB is a Python package (RRID:SCR_008394, version 3.8+) that can be installed on any device where Python is available (pip install bsb) and is open source with source code documentation and topical tutorials. It includes workflows and building tools for multiscale modeling of networks (both reconstruction and simulation) and is compatible with a wide variety of target systems such as personal computers, clusters or supercomputers and provides effortless parallelization using MPI.

Although effective tools for microcircuit modeling have recently appeared [BMTK ${ }^{8,79}$, NetPyNE $\left.{ }^{10}\right]$, their connectivity rules deal well with population-level and probabilistic approaches but a subset of modeling problems remains unsolved, when it comes to dealing with neurons as entities in space with specific morphologies. The BSB addresses these needs with a set of tools designed to work with complex network topologies, cell morphologies and many other spatial and $n$-point problems. These properties allow the BSB to fully empower a "scaffold" modeling strategy, in which a specific brain region or cell type can be modeled and specific cell placement or connectivity datasets can be changed without having to regenerate the entire network.

There are 3 main phases in the scaffold modeling workflow that can be visited iteratively when changes need to be made: configuration, reconstruction and simulation. The core concepts of the framework during the reconstruction phase are i) the network volume, with the definition of various partitions such as layers, meshes or voxel sets (from brain atlases) and arranging elements which can be structured hierarchically to give rise a complex description of the entire region under consideration, ii) the cell types which determine the properties of cell populations, such as their spatial representation (soma radius, geometrical extension and/or morphologies) and density information, iii) the placement of said cell types into subspaces of the network volume using certain placement strategies, and iv) the connectivity between cell types using certain connection strategies. With this information, the framework places and connects the cells, storing the result in a network reconstruction file. Then the simulation phase follows, where cell models, synapse models and devices define the simulator-specific representation of neuron and connection types and input/output variables.

All the above concepts (Fig. 1) can be defined in a preceding configuration phase, either in a configuration file or declared programmatically. The framework was developed with a modular architecture in mind, where each module revolves around a central polymorphic class: the placement module has its PlacementStrategy and the connectivity module its ConnectionStrategy. The users can provide implementations of any interface to extend the repertoire of default placement or connectivity strategies with their own. Each user-defined strategy has access to its configuration node, so users can

374 flexibly configure and parametrize their strategies, leveraging the support provided by the framework.

375 Various other interfaces exist for less commonly extended functions of the framework, such as 
configuration parsers (a JSON parser is provided), simulator backends, storage engines (HDF5 and SONATA backends are provided) or CLI commands.

The scaffold builder compiles its models into HDF5 or SONATA files, a format standard for neural models proposed by the Blue Brain Project and Allen Institute for Brain Science ${ }^{80}$. The HDF5 file is a self-contained hierarchical file that includes all the required information to deploy the model on another machine, including configuration, placement, connectivity and even morphology information. These network architecture files can then be used to reproduce results, optimize parameters or to run entirely different simulations using the same structural information. Parts of the reconstruction can be repeated independently, and the datasets overwritten or appended with the results. This allows for quick tentative changes to be made, which improves iteration times of model development, specific cases and parameter exploration.

\subsubsection{Placement}

The placement is organized into placement objects that consider certain cell types, and a subspace of the volume. These objects determine the number, position, morphology and orientation of each cell, according to the desired placement strategy. A variety of configuration mechanisms exist to define the number of elements to be placed, such as a fixed count, a specific density (volumetric or planar) or a ratio to the density of another type. Other elements can be instantiated as well, with or without 3D positions for other purposes (e.g., fibers with their somatic origin outside the considered brain circuit). A post-processing step after placement may be enabled, where the elements can be pruned, moved, or labelled (e.g., labelling separate zones with their own connectivity patterns or identifying individuals to be hubs in a modular network). Each morphology can be rotated based on the voxel orientation in which it is placed, and fibers crossing multiple voxels can be bent, in order to follow the surface folding of the region. The main placement strategies are (Fig. 1b):

- Particle placement. The neurons are placed randomly and then checked for collisions, using kdtree partitioning of the 3D space ${ }^{81}$. Colliding particles repel each other, the inertia of the particles is proportional to their radius. It is computationally efficient, yields uniform placement in 3D space, working properly even in irregular shapes, and it can deal with multiple cell types of different size. A pruning step can be enabled to remove cells positioned outside the desired subspace.

- Parallel array placement. The neurons are placed in parallel rows on a desired surface, with a certain angle and specific distances between adjacent cells. A direction-specific jitter can be configured.

- Satellite placement. The neurons are placed near each cell of an associated type (planet cells). Satellite positions are chosen at a random distance within a range based on the radii of the planet cells, so that each planet cell has a certain number of satellite cells around it.

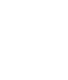




\subsubsection{Connectivity}

Each connection identifies a presynaptic element and a postsynaptic element. When multicompartmental neuron models are used, the synaptic locations on specific morphology compartments are also identified. Connections may target either populations, subpopulations, or only specific regions of the cell morphologies. A post-processing step after connectivity may be enabled, where the identified synapse locations can be re-distributed (e.g. pruning or moving the synapses). The use of cell morphologies can be combined with soma-only approaches. Multiple synapses per pair can be requested, following a probability distribution.

The main connectivity strategies provided by the BSB implement different proximityprobability rules (Fig. 1c):

- $\quad$ Touch detection. The 3D space is partitioned using a kd-tree to search for potentially intersecting cell pairs. Then, the actual points of intersection are determined using another kd-tree specific to the pair morphologies, with a maximum intersection distance parameter.

- Voxel intersection. Each presynaptic cell is represented by a voxelized morphology and these voxels are tested for intersections with the voxels of the postsynaptic cells, using R-tree 3D space partitioning. When matching voxels are found, random compartments in each voxel are selected, introducing variability. It is, in this respect, less deterministic than the touch detection strategy ${ }^{82}$. A Fiber intersection variant exists to optimize the case of long, thin neurites, whose path can be deformed through space according to a 3D field of direction vectors.

- Distance-based in/out degree. A probability distribution is applied to the distance between cells. Optionally 2 additional probability distributions can be given for the indegree and outdegree distribution of the network. The number of postsynaptic elements per presynaptic element is determined by samples of the outdegree distribution. Each postsynaptic target is weighted according to its distance probability, and the probability to transition from their current indegree $\mathrm{N}$ to indegree $\mathrm{N}+1$, as dictated by the indegree distribution. To optimize the algorithm, a kd-tree is queried for cells within a maximum search radius derived from the cumulative probability function of the distance distribution.

\subsubsection{Simulation}

The BSB can instruct simulators to run the configured models. Although multiple adapters to different simulators are provided (NEURON - RRID:SCR_005393; NEST - RRID:SCR_002963; Arbor - 10.5281/zenodo.4428108), there is no common high-level language to send instructions across simulators. Instead, sets of simulator-specific configuration expose the simulator underlying APIs more directly. These classes contain the simulator-specific logic to fully define inputs, execute, monitor progress, and collect output of simulations. The interface to the NEURON simulator has been applied specifically in this work. 
NEURON ${ }^{83}$ cooperates with our Python packages: Arborize to create high-level descriptions of cell models [https://github.com/dbbs-lab/arborize], Patch to provide a convenience layer on top of

451 NEURON [https://github.com/Helveg/patch], and Glia to manage NMODL file dependencies and versioning [https://github.com/dbbs-lab/glia]. Together, these packages and the NEURON adapter provide out-of-the-box load balanced parallel simulations in NEURON. The adapter is capable of creating and connecting these arborized cell models over multiple cores, implements device models such as spike generators, voltage and synapse recorders and collects the recorded measurements in an HDF5 result file. The recorders can specify targets at the cellular or subcellular level, recording membrane or synapse voltages, conductances, currents and ionic concentrations. These easy configurable devices allow to monitor all signals propagating across the network to reproduce results at multiple scales.

\subsubsection{Visualization}

The BSB provides a plotting module to directly visualize simulation results including 3D network plots, cell activity in 3D space, PSTH, raster plots, synaptic currents mapped on cell morphologies, and more. The BSB provides a Blender module containing a complete blender pipeline for rendering videos of the network activity on a single machine or a cluster. The BSB can be used in Blender Python environment and provides functions to synchronize the state of the network with the Blender scene, to animate results or to generate debug frames, to troubleshoot placement, connectivity or simulation issues.

\subsection{The cerebellar cortical model}

Using the BSB, a mouse cerebellar cortical microcircuit was reconstructed and simulated. The example reported here refers to a volume partitioned into a granular, Purkinje and molecular layer. Specifically, the volume extended $300 \mu \mathrm{m}$ along $x$, $200 \mu \mathrm{m}$ along $z$, and $295 \mu \mathrm{m}$ along $y(y=$ layer thickness; $130 \mu \mathrm{m}$ granular layer, $15 \mu \mathrm{m}$ Purkinje cell layer, $150 \mu \mathrm{m}$ molecular layer). In the reference system, $x-y$ is the sagittal plane, $x-z$ the horizontal plane, $z-y$ the coronal plane. The reconstructed volume was $17.7 \cdot 10^{-3} \mathrm{~mm}^{3}$. The model was filled with biophysically detailed compartmental neurons for each cell type. Some structural data and multiple observations from electrical recordings in vivo and in vitro were used as constraints in building the model, further experimental measurements were used for structural and functional validation.

\subsubsection{Neuron placement}

Both the granular layer and the molecular layer were filled using particle placement. The granular layer is made up of densely packed granule cells $(\mathrm{GrC})$ and glomeruli ( $\mathrm{glom}$ ) intercalated with Golgi cells $(G o C)$. Furthermore, a certain number of mossy fibers ( $m f$ ) was created (without any 3D 
position), each terminating in about 20 glomeruli. Each $G r C$ emits an ascending axon ( $a a$ ) that raises perpendicularly to the overlying cerebellar surface and reaches the molecular layer bifurcating into two opposite branches of a parallel fiber ( $p f$ ) elongating on the $z$-axis (major lamellar axis).

The molecular layer was divided into a superficial sublayer ( $2 / 3$ of the thickness) hosting the stellate cells $(S C)$ and a deep sublayer (1/3 of the thickness) hosting the basket cells $(B C)^{23,84}$.

The Purkinje cells $(P C)$ were placed on a horizontal plane $(x-z)$ using parallel array placement. $P C$ s were placed along parallel lines, with an inclination angle of about $70^{\circ}$ with respect to the major lamellar axis. The dendritic tree of the $P C$ is flattened on the sagittal plane and extends for about 150 $\mu \mathrm{m}{ }^{18}$. The parallel arrays were placed at such a distance that the $P C$ dendritic trees did not overlap, while along the major lamellar axis their somata could be packed closely together. For each neuronal population, the nearest neighbour, the pairwise distance distribution, and the radial distribution function were computed.

\subsubsection{Knowledge base for microcircuit connectivity}

This chapter summarizes the fundamental knowledge used to reconstruct cerebellar microcircuit connectivity and highlights which parameters are reported or absent in literature, implying the different strategies adopted in the BSB.

The connectivity of $m f \mathrm{~s}$ and gloms is supported by an extended anatomo-physiological analysis indicating that (i) each $m f$ spreads the input signal into a cluster of $g l o m s{ }^{24}$, (ii) each $G r C$ sends its 4 dendrites to gloms belonging to different $m f \mathrm{~s}$ within about $30 \mu \mathrm{m}^{25}$, (iii) 1 excitatory synapse is formed on the terminal compartment of each $\mathrm{GrC}$ dendrite ${ }^{26,27}$, (iv) 1 excitatory synapse is formed on $\mathrm{GoC}$ basolateral dendrites ${ }^{30}$, (v) 1 inhibitory synapse is formed on the preterminal compartment of each $\mathrm{GrC}$ dendrite ${ }^{85}$.

The connectivity of GoCs is also supported by a robust experimental dataset. (i) GoCs receive an undetermined number of excitatory inputs from $m f s$ through gloms, that in turn host $\sim 2$ GoC basolateral dendrites each ${ }^{30}$. (ii) Given that $G o C$ - $G r C$ synapses are inside $g l o m s$, each $G r C$ dendrite receives inhibition from a $G o C$ whose axon reaches the glom contacting that dendrite ${ }^{28,29}$. (iii) $G o C s$ may receive as many as $\sim 400 a a$ synapses on basolateral dendrites and $\sim 1200 \mathrm{pf}$ synapses on apical dendrites ${ }^{31}$. (iv) GoCs make GABAergic synapses onto other $G o C s{ }^{32}$, but their number was not reported. (v) There are 2-4 gap junctions per GoC pair ${ }^{33}$. Therefore, geometrical rules were used to extract the missing parameters.

The connectivity of $P C$ s can be derived by the axons intercepting their dendritic tree. (i) There is one single synapse per $p f-P C$ crossing. As a whole, the number of $p f-P C$ synapses may range up to $\sim 100.000^{34}$, many of which would be silent ${ }^{86}$. Based on spines density ${ }^{87}$ and the total length of a $P C$ dendritic tree ${ }^{18}$, the number of possible $p f$ synapses was estimated to be $15^{\circ} 000-20^{\circ} 000$, with a 
minimum of $\sim 100$ synapses needed to generate a simple spike ${ }^{35}$. (ii) The number of $a a-P C$ synapses is not known but there would be multiple synapses per $a a-P C$ pair ${ }^{35}$.

The connectivity of $M L I$ is not completely defined. It is known that (i) $M L I s$ receive excitatory input from $p f \mathrm{~s}$, (ii) $M L I \mathrm{~s}$ form inhibitory connections with other $M L I \mathrm{~s}$ of the same type ${ }^{36}$, (iii) collaterals of a $S C$ axon mainly extend on the coronal plane, while collaterals of a $B C$ axon mainly extend on the horizontal plane, innervating multiple $P C \mathrm{~s}^{37}$, (iv) each $P C$ receives 3-50 baskets around the soma from as many different $B C \mathrm{~s}^{38}$, while $S C$ axons terminate on intermediate $P C$ dendritic branches with 0.3-1.6 $\mu \mathrm{m}$ diameter ${ }^{37,59,61}$.

\subsubsection{Selection of microcircuit connectivity rules}

The connectome of the cerebellar network took into consideration 16 connection types (identified by their source and target neuronal population): $m f$-glom, glom-GrC, glom-GoC, GoC-GrC, $G o C-G o C, G r C(a a)-G o C, G r C(a a)-P C, G r C(p f)-G o C, G r C(p f)-P C, G r C(p f)-S C, G r C(p f)-B C, S C-P C$, $B C-P C, S C-S C, B C-B C, G o C-G o C, G o C-G o C$ gap junctions.

Glomerular connectivity is a special case since it is largely constrained by prescribed neuroanatomical and neurophysiological information. Then, since the glom did not have a defined morphological model, they were connected through probability strategies (distance-based in/out degree) to identify nearby compartments for synaptic locations on the target cell types, for which a realistic morphology was used.

Specifically, glomerular connectivity was largely based on prescribed anatomo-physiological rules:

- $\quad m f$-glom. The $m f$ arborization creates anisotropic clusters of glomeruli and clusters originating from different $m f$ s mixed up with each other to some degree ${ }^{24}$. Taking into account short branches since the small reconstructed volume, a local branching algorithm grouped glomeruli ( $20 \pm 3$ per cluster, normally distributed) receiving signals from the same $m f$ by a distance-based probability rule.

- glom-GrC. For each $\operatorname{GrC}$, a pool of nearby gloms were selected based on the distance between the glom barycentre and the $\operatorname{GrC}$ soma center, and a maximum extension of $\mathrm{GrC}$ dendrites of 30-40 $\mu \mathrm{m}{ }^{27}$. From the pool, 4 gloms, each from a different cluster, were randomly sampled and connected with one of the $4 \mathrm{GrC}$ dendrites.

- glom-GoC. For each glom, all GoCs with their soma at a radial distance less than $50 \mu \mathrm{m}$ (corresponding to an average extension of $G o C$ basolateral dendrites, isotropically in $3 \mathrm{D}^{14,31}$ ) were connected. The synapse was placed on a basolateral dendrite using an exponential distribution favouring the compartments closer to the centre of the glom.

- GoC-GrC. This connection absorbed the connection GoC-glom which was generated using 3D proximity and a mean divergence (out-degree) of 40; then, each $G o C$ synapsed directly on all $\mathrm{GrCs}$ that shared those gloms. 
The rest of the cerebellar connectome was reconstructed by applying voxel and fiber intersection, and touch detection. Voxel intersection was preferred when 3D morphologies were intersecting. This strategy, by introducing a cubic convolution and randomization, reduced overfitting artifacts arising from the intersection of identical morphologies arranged in the quasi-crystalline cerebellar microcircuit. Touch detection and fiber intersection were preferred when dealing with 2D fibers (i.e. $a a$ and $p f$ ), for which the voxel intersection would create cubic volumes not representative of the fibers as line segments. Therefore, for the connections involving aas $(\operatorname{GrC}(a a)-G o C$ and $\operatorname{GrC}(a a)-P C)$, touch detection was applied with a tolerance distance of $3 \mu \mathrm{m}$. For the connections involving $p f \mathrm{~s}(\operatorname{GrC}(p f)-G o C, \operatorname{GrC}(p f)-P C, \operatorname{GrC}(p f)-S C, \operatorname{GrC}(p f)-B C)$, fiber intersection was applied with affinity $=0.1$ and resolution $=20 \mu \mathrm{m}$. For the other connections, voxel intersection was applied: $G o C-G o C, S C-S C$, and $B C-B C$ with affinity $=0.5 ; S C-P C$ with affinity $=0.1 ; B C-P C$ with affinity $=1$, $G o C-G o C$ gap junctions with affinity= 0.2. For chemical synapses, the presynaptic compartment was always axonal and the postsynaptic compartment was dendritic or somatic (as for the $B C-P C$ connection). For electrical synapses, gap junctions were created between dendrites. Following biological indications, specific sectors of morphologies were selected as source or target for synaptic localization, and, for each connected cell pair, the desired number of synapses was defined, eventually as a normal distribution (mean $\pm \mathrm{sd}$ ) (see table in Fig. 2d).

Specific sectors of the dendritic trees of $G o C$ and $P C$ were used as targets for synapse formation. $G o C$ s receive inhibitory and electrical synapses from other $G o C$ s as well as $a a$ synapses on basolateral dendrites in the granular layer, while $p f$ synapses impinge on apical dendrites in the molecular layer ${ }^{31}$. $P C$ s receive $a a$ and $p f$ synaptic inputs on different parts of the dendritic tree, and also $S C \mathrm{~s}$ and $B C \mathrm{~s}$ target different parts of the neuron ${ }^{59,61}$. (i) $G r C(a a) \rightarrow P C$ dendrites with diameter $<0.75 \mu \mathrm{m}^{18,39}$. (ii) $G r C(p f) \rightarrow P C$ dendrites with diameter between 0.75 and $1.6 \mu \mathrm{m}$. (iii) $S C \rightarrow P C$ dendrites with diameter between 0.3 and $1.6 \mu \mathrm{m}^{37,59,61}$. (iv) $B C \rightarrow P C$ soma.

\subsubsection{Multi-compartmental neuron models and synaptic models}

Detailed multi-compartmental models of $G r C, G o C, P C, S C, B C$ are available, in which dendritic and axonal processes are endowed with voltage-dependent ionic channels and synaptic receptors. In each model, cell-specific aspects critical for function are reproduced, e.g., the role of the axon initial segment, spontaneous firing and burst-pause behaviour. The following receptor-channel models, all validated against in vitro recordings, and generic gap junction models were inserted in the appropriate neuron compartments:

- $G r C$ synapses ${ }^{17,88} . m f$-GrC: AMPA and NMDA receptors; $G o C$ - $G r C$ : GABAalpha1/6 receptors.

- $\quad G o C$ synapses ${ }^{16}$. pf-GoC: AMPA; $a a-G o C$ : AMPA and NMDA; $m f-G o C$ : AMPA and NMDA; GoC-GoC: GABAalpha1, gap junctions ${ }^{33,89}$.

- $\quad P C(\mathrm{Z}+$ type $)$ synapses $^{59} \cdot p f-P C$ and $a a-P C$ : AMPA; $S C-P C$ and $B C-P C$ : GABAalpha1.

- $\quad S C$ and $B C$ synapses ${ }^{19} . p f-S C$ and $p f-B C$ : AMPA and NMDA; $S C-S C$ and $B C-B C$ : GABAalpha1. 
Chemical neurotransmission was modelled using the Tzodyks and Markram scheme ${ }^{90,91}$ for neurotransmitter release, and receptors kinetic schemes for postsynaptic receptor activation.

595 Glutamatergic neurotransmission could activate either only AMPA or both AMPA and NMDA receptors ${ }^{92}$. GABAergic neurotransmission activated GABA-A receptors ${ }^{93}$. A neurotransmitter impulse was followed by a slow diffusion wave generating both a transient and a sustained component of the postsynaptic response, as observed experimentally. Parameters describing release probability, diffusion, ionic receptor mechanisms, vesicle cycling, recovery time constant, electrical conduction were derived from the corresponding original papers.

601

\subsubsection{Network simulations: stimulation and analysis}

All simulations used the NEURON adapter of the BSB and were run in parallel through MPI on the CSCS Piz Daint supercomputer, with a time resolution of $0.025 \mathrm{~ms}$. Simulations started with a 5-s stabilization period followed by a $100 \mathrm{~ms}$ initialization period, in which random $\mathrm{mf}$ inputs desynchronized the network. In all simulations, spikes and voltage traces at soma of all neurons were recorded. Depending on specific analyses, in some simulations further microscopic variables were recorded, as explained below. A set of stimulation protocols reproducing specific spatio-temporal patterns of $m f$ activity was used to functionally validate the cerebellar network model; in some cases, the protocols were repeated using an altered version of the network model in terms of connectome ("bylesion" approach), to quantitatively check the relative roles of the connection types.

\subsubsection{Diffused background stimulation}

The cerebellum in vivo is constantly bombarded by a diffused background noise, which determines the resting state activity of neurons and is thought to entrain the network into coherent lowfrequency oscillations ${ }^{46,55,56}$. Therefore, we first explored the response of the network model to a random Poisson noise at $4 \mathrm{~Hz}^{20}$ on all $m f$ s for 4 seconds, proving a testbench to validate the structural and functional network balance.

- Steady state analysis. We compared basal discharges in the network to those recorded at rest in vivo. The mean frequency of each population was computed.

- Oscillatory state analysis. We investigated the emergence of low-frequency coherent oscillations in the $G o C$ and $G r C$ populations. The power spectrum of $G o C$ and $G r C$ firing activity was computed by Fast Fourier Transform (FFT), applied to time-binned spike-counts ( $2.5 \mathrm{~ms}$ bins). The zero-component was cut off and the FFT was smoothed using a Savitzki-Golay filter (6th order polynomial, window of 51 bins). The same analysis was performed when $G o C$-GoC gap junctions were blocked, in order to check the role of electrical coupling in oscillatory behaviour of the granular layer. 


\subsubsection{2 mf burst stimulation}

The cerebellum in vivo responds with localized burst-burst patterns to facial or whisker sensory stimulation ${ }^{20,51}$. These bursts are supposed to run on collimated $m f$ bundles generating dense response clusters in the granular layer and thereby activating the neuronal network downstream ${ }^{21,22,49,88}$. To simulate this functional response, we delivered a $m f$ stimulus burst, superimposed on background noise at $4 \mathrm{~Hz}$, to $4 m f \mathrm{~s}$ in the center of the horizontal plane, activating about 80 gloms. The $m f$ burst lasted 20 ms and was made of 5 spikes at fixed time instants (on average $200 \mathrm{~Hz}$, maximum $250 \mathrm{~Hz}$ ), within range of in vivo patterns ${ }^{20,39,51}$. Ten simulations were run to account for random variability of the background input and the network responses. Multiple variables over time were recorded: spike times and membrane voltages of every cell, synaptic currents in the dendrites of some cells, the internal calcium concentration $\left[\mathrm{Ca}^{2+}\right]_{\text {in }}$ in the dendrites of all $\mathrm{GrCs}$ and of some $\mathrm{GoCs}$. Further simulations were carried out using different values of neurotransmitter release probability at the $m f$ - $G r C$ synapse (from $\mathrm{u}=0.43$ to $\mathrm{u}=0.1$ and to $\mathrm{u}=0.9)$.

- General analysis of response patterns. For each neuronal population, a raster plot and a PSTH (peristimulus time histogram) was computed. Each population was described using Multiple Regression Analysis: the dependent variable was the average firing frequency during $40 \mathrm{~ms}$ after the stimulus onset, over 10 simulations, and the independent variables were the average numbers of spikes received from each presynaptic population. The linear regression was reported as direction coefficients and $\mathrm{R}^{2}$ score.

- Analysis of granular layer responses. For GrCs we related the number of dendrites activated by the $m f$ burst with the number of output spikes and the first spike latency. The same protocol was carried out while switching-off phasic and tonic inhibition from GoCs (GABA-A receptor blockade). This allowed us to investigate excitatory-inhibitory loops in the granular layer, by estimating the response patterns of $\mathrm{GrCs}$, their latencies, and the fraction of $\mathrm{GrCs}$ activated compared with the control condition. Furthermore, for each $\mathrm{GrC}$, the level of $\left[\mathrm{Ca}^{2+}\right]_{\text {in }}$ in the dendrites averaged on 500 ms from the $m f$ burst was extracted and these $\left[\mathrm{Ca}^{2+}\right]_{\text {in }}$ values were related to the number of dendrites activated by the stimulus. The correlation analysis used Normalized Mutual Information (NMI) ${ }^{94}$. - Analysis of PC responses. The $P C$ response was analyzed to evaluate the burst-pause behavior. For each $P C$, an automatic algorithm extracted any shortening of the inter-spike intervals during the stimulus window (burst coeff.) and any elongation after the stimulus (pause coeff.) compared to baseline. The burst coeff. was correlated with the number of excitatory synaptic inputs (from $p f \mathrm{~s}$ and aas) by multiple regression analysis. The pause coeff. was correlated with the number of inhibitory synaptic inputs (from $M L I \mathrm{~s}$ ) received during the burst stimulation (20 ms $m f$ burst +20 ms of delayed effects), by calculating the Normalized Mutual Information (NMI). Furthermore, the relation between the burst and pause coefficients themselves was analysed, by NMI. Further simulations were run clamping an on-beam $P C$ at $-70 \mathrm{mV}$, recording all synaptic currents. All excitatory synaptic currents (AMPA from $a a s$ and $p f s$ ) and all inhibitory synaptic currents (GABA 
from $S C \mathrm{~s}$ and $B C \mathrm{~s}$ ) were summed up, then the cross-correlation among these two rectified and normalized currents was calculated, to identify the time lag between them.

- Visualization of subcellular variables. In some cases, ad-hoc computationally expensive recordings of multiple microscopic variables were performed. In an example focused on a $G o C$, all synaptic currents (AMPA and NMDA from gloms on basolateral dendrites, AMPA and NMDA from aas on basolateral dendrites, AMPA from $p f s$ on apical dendrites, GABA from other GoCs on basolateral dendrites), and $\left[\mathrm{Ca}^{2+}\right]_{\text {in }}$ were recorded and animated (see Visualization).

673

\subsubsection{Lateral Poisson stimulation}

The lateral inhibition from $M L I$ s to $P C$ s comes from activated $M L I$ s providing inhibition to off676 beam $P C$ s, mainly from $B C$ s due to their axon orientation ${ }^{43}$. To simulate this functional response, we 677 delivered a $50 \mathrm{~Hz}$ Poisson distributed stimulus, lasting $100 \mathrm{~ms}$, superimposed on the background noise 678 (at $4 \mathrm{~Hz}$ ), on $24 \mathrm{mfs}$ on one side of the volume, to monitor the modulation of $M L I$ inhibitory effects on $679 P C$ s at different distances from the active cluster. Two conditions were evaluated: i) control and ii) 680 MLIs disconnected from PCs. Ten simulations for each condition were carried out.

681 - Analysis of PC responses. For each PC, the average Inter-Spike-Interval (ISI) during $200 \mathrm{~ms}$ 682 baseline and the average ISI during the 100-ms stimulus was computed. The relationship between 683 the distance of a $P C$ from the active cluster and its activity modulation (balance between $\mathrm{GrCs}$ 684 excitation and MLIs inhibition) was investigated in control condition and in the "no MLI-PC" condition. 
The BSB source code is available at https://github.com/dbbs-lab/bsb, and can be installed through pip as a Python package available at https://pypi.org/project/bsb/. Documentation can be found at https://bsb.readthedocs.io/.

693

1. Arbib, M. A. \& Érdi, P. Precis of neural organization: Structure, function, and dynamics.

2. D'Angelo, E. \& Gandini Wheeler-Kingshott, C. Modelling the brain: Elementary components to explain ensemble functions. Riv. del nuovo Cim. 40, 273-333 (2017).

3. Amunts, K. et al. The Human Brain Project—Synergy between neuroscience, computing, informatics, and brain-inspired technologies. PLOS Biol. 17, e3000344 (2019).

4. Markram, H. et al. Reconstruction and Simulation of Neocortical Microcircuitry. Cell 163, 456-492 (2015).

5. Brette, R. et al. Simulation of networks of spiking neurons: A review of tools and strategies. $J$. Comput. Neurosci. 23, 349-398 (2007).

6. Hines, M. L. \& Carnevale, N. T. The NEURON Simulation Environment. Neural Comput. 9, 1179-1209 (1997).

7. Gewaltig, M.-O. \& Diesmann, M. NEST (NEural Simulation Tool). Scholarpedia 2, 1430 (2007).

8. Dai, K. et al. Brain Modeling ToolKit: An open source software suite for multiscale modeling of brain circuits. PLoS Comput. Biol. 16, e1008386 (2020).

9. Gratiy, S. L. et al. BioNet: A Python interface to NEURON for modeling large-scale networks. PLoS One 13, 1-18 (2018).

10. Dura-Bernal, S. et al. NetpyNE, a tool for data-driven multiscale modeling of brain circuits. Elife 8, 1-26 (2019).

715 11. Marr, D. A theory of cerebellar cortex. J. Physiol. 202, 437-470 (1969).

12. D'Angelo, E. et al. Modeling the cerebellar microcircuit: New strategies for a long-standing issue. Frontiers in Cellular Neuroscience 10, 176 (2016). 
14. Solinas, S., Nieus, T. \& D'Angelo, E. A realistic large-scale model of the cerebellum granular layer predicts circuit spatio-temporal filtering properties. Front. Cell. Neurosci. 4, 12 (2010).

15. Casali, S., Tognolina, M., Gandolfi, D., Mapelli, J. \& D’Angelo, E. Cellular-resolution mapping uncovers spatial adaptive filtering at the rat cerebellum input stage. Commun. Biol. 3,

16. Masoli, S., Ottaviani, A., Casali, S. \& D’Angelo, E. Cerebellar Golgi cell models predict dendritic processing and mechanisms of synaptic plasticity. PLoS Comput. Biol. 16, e1007937

17. Masoli, S., Tognolina, M., Laforenza, U., Moccia, F. \& D’Angelo, E. Parameter tuning

19. Rizza, M. et al. Stellate cell computational modeling predicts signal filtering in the molecular layer circuit of cerebellum. Sci. Rep. 11, (2021).

21. Roggeri, L., Rivieccio, B., Rossi, P. \& D’Angelo, E. Tactile stimulation evokes long-term

22. Ramakrishnan, K. B., Voges, K., De Propris, L., De Zeeuw, C. I. \& D’Angelo, E. Tactile

23. Sultan, F. \& Bower, J. M. Quantitative golgi study of the rat cerebellar molecular layer

24. Sultan, F. Distribution of mossy fibre rosettes in the cerebellum of cat and mice: evidence for a Cerebellar Input Layer Enables Lossless Sparse Encoding. Neuron 83, 960-974 (2014).

6. Jakab, R. L. \& Hámori, J. Quantitative morphology and synaptology of cerebellar glomeruli in 
the rat. Anat. Embryol. (Berl). 179, 81-8 (1988).

751

27. Houston, C. M. et al. Exploring the significance of morphological diversity for cerebellar granule cell excitability. Sci. Rep. 7, 46147 (2017).

28. Hamori, J., Jakab, R. L. \& Takacs, J. Morphogenetic plasticity of neuronal elements in cerebellar glomeruli during deafferentation-induced synaptic reorganization. J. Neural Transplant. Plast. 6, 11-20 (1997).

29. Mapelli, L., Solinas, S. \& D'Angelo, E. Integration and regulation of glomerular inhibition in the cerebellar granular layer circuit. Front. Cell. Neurosci. 8, 55 (2014).

30. Tabuchi, S., Gilmer, J. I., Purba, K. \& Person, A. L. Pathway-specific drive of cerebellar golgi cells reveals integrative rules of cortical inhibition. J. Neurosci. 39, 1169-1181 (2019).

31. Cesana, E. et al. Granule Cell Ascending Axon Excitatory Synapses onto Golgi Cells Implement a Potent Feedback Circuit in the Cerebellar Granular Layer. J. Neurosci. 33, 12430-12446 (2013).

32. Hull, C. \& Regehr, W. G. Identification of an Inhibitory Circuit that Regulates Cerebellar Golgi Cell Activity. Neuron 73, 149-158 (2012).

33. Szoboszlay, M. et al. Functional Properties of Dendritic Gap Junctions in Cerebellar Golgi Cells. Neuron 90, 1043-1056 (2016).

34. Hoxha, E., Tempia, F., Lippiello, P. \& Miniaci, M. C. Modulation, plasticity and pathophysiology of the parallel fiber-purkinje cell synapse. Front. Synaptic Neurosci. 8, 1-16 (2016).

35. Walter, J. T. \& Khodakhah, K. The linear computational algorithm of cerebellar Purkinje cells. J. Neurosci. 26, 12861-12872 (2006).

36. Kondo, S. \& Marty, A. Synaptic currents at individual connections among stellate cells in rat cerebellar slices. J. Physiol. 509 ( Pt 1, 221-32 (1998).

37. Ango, F. et al. Bergmann Glia and the Recognition Molecule CHL1 Organize GABAergic Axons and Direct Innervation of Purkinje Cell Dendrites. PLoS Biol. 6, e103 (2008).

38. Blot, A. \& Barbour, B. Ultra-rapid axon-axon ephaptic inhibition of cerebellar Purkinje cells by the pinceau. Nat. Neurosci. 17, 289-295 (2014).

39. Wilms, C. D. \& Häusser, M. Reading out a spatiotemporal population code by imaging neighbouring parallel fibre axons in vivo. Nat. Commun. 6, 1-9 (2015).

40. Solinas, S. et al. Fast-reset of pacemaking and theta-frequency resonance patterns in cerebellar 
41. Forti, L., Cesana, E., Mapelli, J. \& D’Angelo, E. Ionic mechanisms of autorhythmic firing in rat cerebellar Golgi cells. J. Physiol. 574, 711-729 (2006).

42. Arancillo, M., White, J. J., Lin, T., Stay, T. L. \& Sillitoe, R. V. In vivo analysis of purkinje cell firing properties during postnatal mouse development. J. Neurophysiol. 113, 578-591 (2015).

43. Kim, J. \& Augustine, G. J. Molecular Layer Interneurons: Key Elements of Cerebellar Network Computation and Behavior. Neuroscience (2020). doi:10.1016/j.neuroscience.2020.10.008

44. Jirenhed, D.-A., Bengtsson, F. \& Jörntell, H. Parallel fiber and climbing fiber responses in rat cerebellar cortical neurons in vivo. Front. Syst. Neurosci. 7, 16 (2013).

45. Barmack, N. H. \& Yakhnitsa, V. Functions of interneurons in mouse cerebellum. J. Neurosci. 28, 1140-1152 (2008).

46. Hartmann, M. J. \& Bower, J. M. Oscillatory activity in the cerebellar hemispheres of unrestrained rats. J. Neurophysiol. 80, 1598-1604 (1998).

47. Dugué, G. P. et al. Electrical Coupling Mediates Tunable Low-Frequency Oscillations and Resonance in the Cerebellar Golgi Cell Network. Neuron 61, 126-139 (2009).

48. D'Angelo, E., De Filippi, G., Rossi, P. \& Taglietti, V. Synaptic excitation of individual rat cerebellar granule cells in situ: evidence for the role of NMDA receptors. J. Physiol. 484, 397413 (1995).

49. Diwakar, S., Lombardo, P., Solinas, S., Naldi, G. \& D’Angelo, E. Local field potential modeling predicts dense activation in cerebellar granule cells clusters under LTP and LTD control. PLoS One 6, 21928 (2011).

50. Eccles, J. C., Ito, M. \& Szentágothai, J. The Cerebellum as a Neuronal Machine. The Cerebellum as a Neuronal Machine (Springer Berlin Heidelberg, 1967). doi:10.1007/978-3$662-13147-3$

51. Chadderton, P., Margie, T. W. \& Häusser, M. Integration of quanta in cerebellar granule cells during sensory processing. Nature 428, 856-860 (2004).

52. Arenz, A., Silver, R. A., Schaefer, A. T. \& Margrie, T. W. The contribution of single synapses to sensory representation in vivo. Science (80-. ). 321, 977-980 (2008).

53. Powell, K., Mathy, A., Duguid, I. \& Häusser, M. Synaptic representation of locomotion in 
single cerebellar granule cells. Elife 4, (2015).

54. Ishikawa, T., Shimuta, M. \& Häuser, M. Multimodal sensory integration in single cerebellar granule cells in vivo. Elife 4, (2015).

55. Vos, B. P., Volny-Luraghi, A. \& De Schutter, E. Cerebellar Golgi cells in the rat: Receptive fields and timing of responses to facial stimulation. Eur. J. Neurosci. 11, 2621-2634 (1999).

56. Solinas, S. M. G., Maex, R. \& De Schutter, E. Dendritic amplification of inhibitory postsynaptic potentials in a model Purkinje cell. Eur. J. Neurosci. 23, 1207-1218 (2006).

57. Holtzman, T., Rajapaksa, T., Mostofi, A. \& Edgley, S. A. Different responses of rat cerebellar Purkinje cells and Golgi cells evoked by widespread convergent sensory inputs. J. Physiol. 574, 491-507 (2006).

58. Herzfeld, D. J., Kojima, Y., Soetedjo, R. \& Shadmehr, R. Encoding of action by the Purkinje cells of the cerebellum. Nature 526, 439-441 (2015).

59. Masoli, S. et al. Single neuron optimization as a basis for accurate biophysical modeling: The case of cerebellar granule cells. Front. Cell. Neurosci. 11, 71 (2017).

60. Bower, J. M. \& Woolston, D. C. Congruence of spatial organization of tactile projections to granule cell and Purkinje cell layers of cerebellar hemispheres of the albino rat: vertical organization of cerebellar cortex. J. Neurophysiol. 49, 745-766 (1983).

61. Lu, H., Esquivel, A. V. \& Bower, J. M. 3D electron microscopic reconstruction of segments of rat cerebellar Purkinje cell dendrites receiving ascending and parallel fiber granule cell synaptic inputs. J. Comp. Neurol. 514, 583-594 (2009).

62. Gundappa-Sulur, G., De Schutter, E. \& Bower, J. M. Ascending granule cell axon: An important component of cerebellar cortical circuitry. J. Comp. Neurol. 408, 580-596 (1999).

63. Lennon, W., Hecht-nielsen, R. \& Yamazaki, T. A spiking network model of cerebellar Purkinje cells and molecular layer interneurons exhibiting irregular firing. Front. Comput. Neurosci. 8, 1-10 (2014).

64. Jörntell, H., Bengtsson, F., Schonewille, M. \& De Zeeuw, C. I. Cerebellar molecular layer interneurons - computational properties and roles in learning. Trends Neurosci. 33, 524-535 (2010).

65. Soha, J. M., Kim, S., Crandall, J. E. \& Vogel, M. W. Rapid Growth of Parallel Fibers in the Cerebella of Normal and Staggerer Mutant Mice. J. Comp. Neurol 389, (Wiley-Liss, Inc, 1997). 
66. Zhou, H., Voges, K., Lin, Z., Ju, C. \& Schonewille, M. Differential Purkinje cell simple spike activity and pausing behavior related to cerebellar modules. J. Neurophysiol. 113, 2524-2536 (2015).

67. D'Angelo, E. \& Casali, S. Seeking a unified framework for cerebellar function and dysfunction: from circuit operations to cognition. Front. Neural Circuits 6, 1-23 (2013).

68. Reimann, M. W., King, J. G., Muller, E. B., Ramaswamy, S. \& Markram, H. An algorithm to predict the connectome of neural microcircuits. Front. Comput. Neurosci. 0, 28 (2015).

69. Mapelli, J., Gandolfi, D. \& D`Angelo, E. High-pass filtering and dynamic gain regulation enhance vertical bursts transmission along the mossy fiber pathway of cerebellum. Front. Cell. Neurosci. 0, 14 (2010).

70. Mapelli, J., Gandolfi, D. \& D’Angelo, E. Combinatorial Responses Controlled by Synaptic Inhibition in the Cerebellum Granular Layer. https://doi.org/10.1152/jn.00642.2009 103, 250261 (2010).

71. Apps, R. \& Hawkes, R. Cerebellar cortical organization: a one-map hypothesis. Nat. Rev. Neurosci. 10, 670-681 (2009).

72. Apps, R. et al. Cerebellar Modules and Their Role as Operational Cerebellar Processing Units. The Cerebellum 17, 654-682 (2018).

73. De Zeeuw, C. I. Bidirectional learning in upbound and downbound microzones of the cerebellum. Nat. Rev. Neurosci. 22, 92-110 (2021).

74. K, P. \& NN, U. Intrinsic biophysical diversity decorrelates neuronal firing while increasing information content. Nat. Neurosci. 13, 1276-1282 (2010).

75. Geminiani, A. et al. Complex dynamics in simplified neuronal models: reproducing Golgi cell electroresponsiveness. Front. Neuroinform. 12, 1-19 (2018).

76. Geminiani, A., Casellato, C., D’Angelo, E. \& Pedrocchi, A. Complex electroresponsive dynamics in olivocerebellar neurons represented with extended-generalized leaky integrate and fire models. Front. Comput. Neurosci. 13, (2019).

77. Huang, M., de Koning, T. J., Tijssen, M. A. J. \& Verbeek, D. S. Cross-disease analysis of depression, ataxia and dystonia highlights a role for synaptic plasticity and the cerebellum in the pathophysiology of these comorbid diseases. Biochim. Biophys. Acta-Mol. Basis Dis. 1867, 165976 (2021).

78. Peter, S. et al. Dysfunctional cerebellar Purkinje cells contribute to autism-like behaviour in Shank2 -deficient mice. Nat. Commun. 201671 7, 1-14 (2016). 
79. Billeh, Y. N. et al. Systematic Integration of Structural and Functional Data into Multi-scale Models of Mouse Primary Visual Cortex. Neuron 106, 388-403.e18 (2020).

80. Dai, K. et al. The SONATA Data Format for Efficient Description of Large-Scale Network Models. SSRN Electron. J. 1-24 (2019). doi:10.2139/ssrn.3387685

81. Bentley, J. L. Multidimensional binary search trees used for associative searching. Commun. ACM 18, 509-517 (1975).

82. Nolte, M., Reimann, M. W., King, J. G., Markram, H. \& Muller, E. B. Cortical reliability amid noise and chaos. Nat. Commun. 10, 1-15 (2019).

83. Hines, M. L. \& Carnevale, N. T. Neuron: A Tool for Neuroscientists. Neurosci. 7, 123-135 (2001).

84. Whitney, E. R., Kemper, T. L., Rosene, D. L., Bauman, M. L. \& Blatt, G. J. Density of cerebellar basket and stellate cells in autism: Evidence for a late developmental loss of Purkinje cells. J. Neurosci. Res. 87, 2245-2254 (2009).

85. Mapelli, L., Rossi, P., Nieus, T. \& D’Angelo, E. Tonic Activation of GABA B Receptors Reduces Release Probability at Inhibitory Connections in the Cerebellar Glomerulus. $J$. Neurophysiol. 101, 3089-3099 (2009).

86. Isope, P. \& Barbour, B. Properties of unitary granule cell $\rightarrow$ Purkinje cell synapses in adult rat cerebellar slices. J. Neurosci. 22, 9668-9678 (2002).

87. Parajuli, L. K. \& Koike, M. Three-Dimensional Structure of Dendritic Spines Revealed by Volume Electron Microscopy Techniques. Front. Neuroanat. 15, (2021).

88. Diwakar, S., Magistretti, J., Goldfarb, M., Naldi, G. \& D’Angelo, E. Axonal Na+ channels ensure fast spike activation and back-propagation in cerebellar granule cells. J. Neurophysiol. 101, 519-532 (2009).

89. Vervaeke, K., Lorincz, A., Nusser, Z. \& Silver, R. A. Gap junctions compensate for sublinear dendritic integration in an inhibitory network. Science (80-. ). 335, 1624-1628 (2012).

90. Markram, H. \& Tsodyks, M. Redistribution of synaptic efficacy between neocortical pyramidal neurons. Nature 382, 807-810 (1996).

91. Tsodyks, M. V. \& Markram, H. The neural code between neocortical pyramidal neurons depends on neurotransmitter release probability. Proc. Natl. Acad. Sci. U. S. A. 94, 719-723 (1997).

92. Nieus, T. et al. LTP regulates burst initiation and frequency at mossy fiber-granule cell 


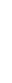

synapses of rat cerebellum: experimental observations and theoretical predictions. $J$. Neurophysiol. 95, 686-99 (2006).

93. Nieus, T. R., Mapelli, L. \& D’Angelo, E. Regulation of output spike patterns by phasic inhibition in cerebellar granule cells. Front. Cell. Neurosci. 8, 246 (2014).

94. Laarne, P., Zaidan, M. A. \& Nieminen, T. ennemi: Non-linear correlation detection with mutual information. SoftwareX 14, 100686 (2021). 


\section{Acknowledgements/funding}

915 This research has received funding from the European Union's Horizon 2020 Framework Program for 916 Research and Innovation under the Specific Grant Agreement No. 945539 (Human Brain Project SGA3) 917 and Specific Grant Agreement No. 785907 (Human Brain Project SGA2) and from Centro Fermi project 918 "Local Neuronal Microcircuits" to ED. Special acknowledgement to EBRAINS and FENIX for 919 informatic support and infrastructure.

920

\section{Author contribution}

$922 \mathrm{CC}$ and RDS designed and developed the informatic framework and performed the simulations; RDS 923 wrote most of the code; AG, SM, MR, AA contributed with essential model components; RDS, CC, 924 ED analyzed the data, wrote the manuscript, and prepared the figures; CC and ED coordinated the work 925 and the EBRAINS interaction. ED promoted the project, supported it financially, defined the 926 physiological aspects and finalized the manuscript.

927

928 10 Conflict of interest:

929 The authors declare that the research was conducted in the absence of any commercial or financial relationships that could be construed as a potential conflict of interest.

\section{Figures and legends}

936 Figures, or their specific panels can be visualized as interactive .html files from https://dbbs-

937 lab.github.io/deschepper-etal-2021/, adding e.g. "figure" + number + panel + ".html" e.g.: 938 "figure2a.html", on this URL. 
Figure 1 | The Brain Scaffold Builder. (a) Core BSB operations. In the reconstruction phase, BSB proceeds by sequentially defining the network volume, cell types, cell placement, cell connectivity. Once neurons and fibers are positioned, their geometries/morphologies are imported and connection rules allow to wire them up and to build the network connectome. In the simulation phase, neuron and synapse models are linked to simulators, like NEURON in the present case, by a specific adapter and interfaced to a set of devices for stimulation and recording. In the post-simulation phase, graphic tools are made available for data representation. This workflow is applicable to any kind of brain neuronal network. (b) Infographic representations of the main placement strategies available in BSB, using kdtree partitioning of the 3D space (particle placement, parallel array placement, satellite placement). (c) Infographic representations of the main connection strategies available in BSB: distance-based in/out degree probability functions, voxel (or fiber) intersection based on voxelization of morphologies, touch detection.

\section{a}
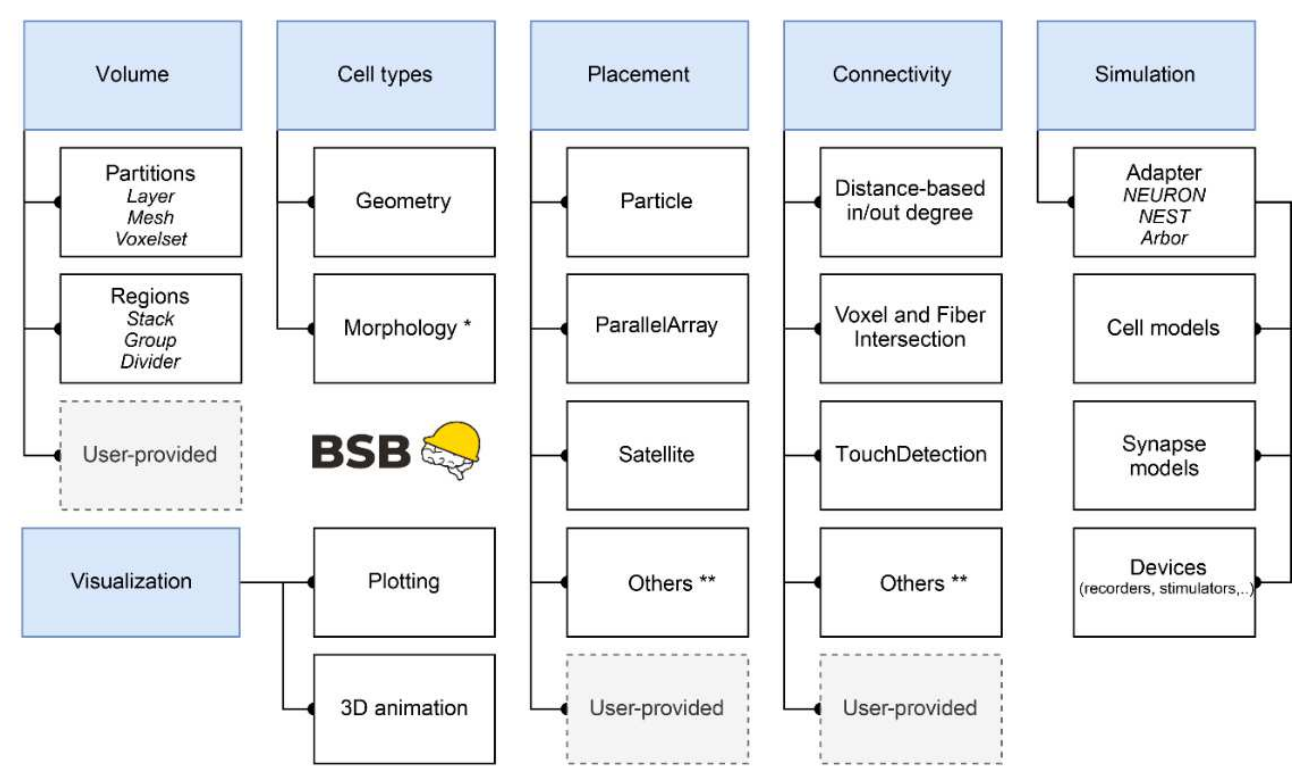

b
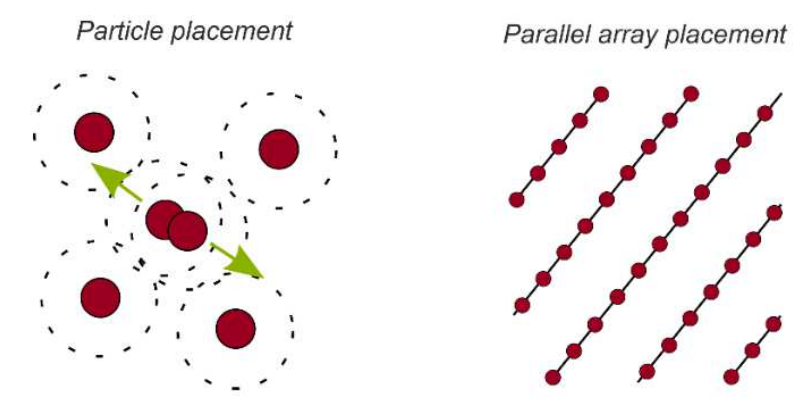

Satellite placement

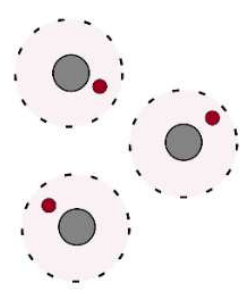

C probability rules

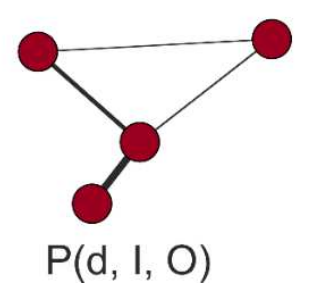

Voxel intersection

Touch detection
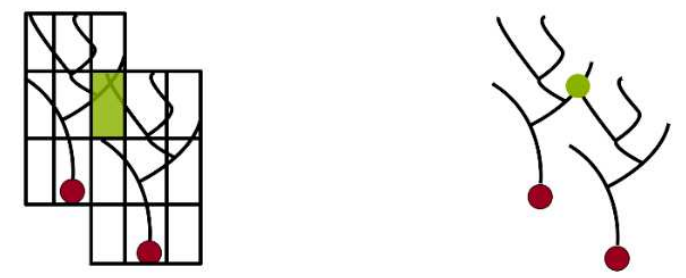
Figure 2 | Reconstruction of the microcircuit of cerebellar cortex. (a) Positioning of cell bodies in a 3D slab $\left(300 \times 295 \times 200 \mu \mathrm{m}^{3}\right)$ of mouse cerebellar cortex. Cell numbers are indicated (the symbols reflect soma size). In this and the following figures, the $x y z$ reference system is defined by $x-y$ (sagittal plane), $x-z$ (horizontal plane), $z-y$ (coronal plane), as in standard anatomical representation. Thus, $y$ measures cortex thickness ( $a$ a direction), while $z$ identifies the major lamellar axis ( $p f$ direction). (b) Example of 3D morphologies illustrating $G r C$ - $G o C$ connections through $a a$ and $p f$. One $G r C$ and two GoCs are shown: the synapse along $a a$ is identified by touch detection, while synapses along $p f$ are identified by fiber intersection. (c) glom-GrC and GoC-GrC connections. A glom contacts a group of $38 \mathrm{GrCs}$ forming an excitatory synapse on the terminal compartment of 1 of their 4 dendrites. The glom, in turn, is contacted by a GoC nearby, which forms an inhibitory synapse on the preterminal dendritic compartment of the same GrCs. The inset shows a $G r C$ with 1 excitatory synapse and 1 inhibitory synapse on each dendrite. (d) The cerebellar cortical connectome generated by BSB reporting convergence (on the postsynaptic element), divergence (from the presynaptic element), total number of synapses, and number of synapses for each connected pair. It should be noted that $\mathrm{mf}$-glom is not a proper synapse but just a branching.
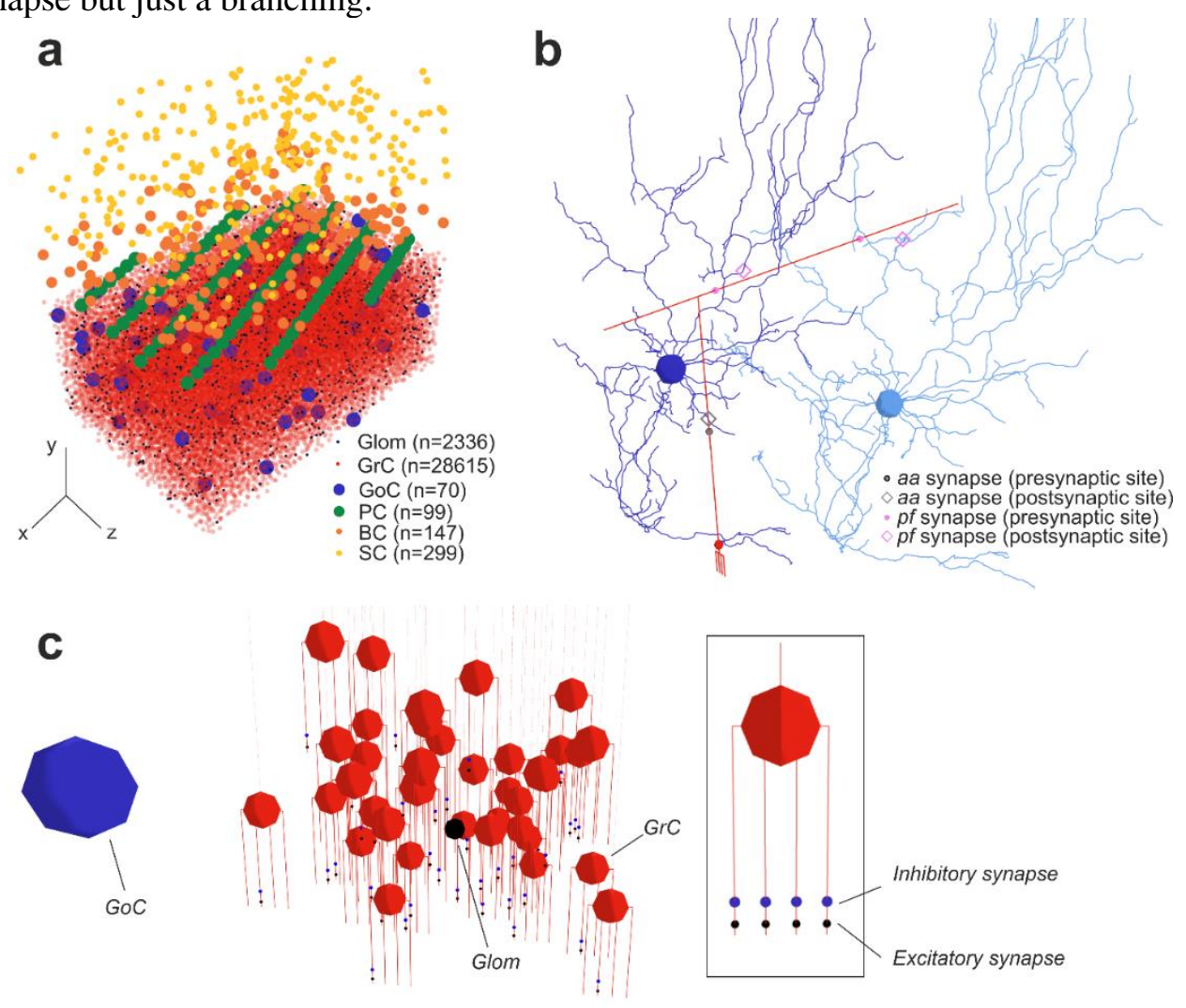

\begin{tabular}{|c|c|c|c|c|}
\hline & Convergence & Divergence & Synapses & Synapses/pair \\
\hline mf-Glom & $1 \pm 0$ & $20 \pm 8$ & 2300 & $1 \pm 0$ \\
\hline Glom-GrC & $4 \pm 0$ & $49 \pm 26$ & 110000 & $1 \pm 0$ \\
\hline Glom-GoC & $56 \pm 21$ & $1.7 \pm 1.4$ & 3900 & $1 \pm 0$ \\
\hline GoC-GrC & $2.4 \pm 0.88$ & $1000 \pm 460$ & 97000 & $1.4 \pm 0.66$ \\
\hline GoC-Glom & $0.84 \pm 0.37$ & $28 \pm 14$ & 2000 & $1 \pm 0$ \\
\hline GoC-GoC & $16 \pm 6.5$ & $16 \pm 7.1$ & 180000 & $160 \pm 5$ \\
\hline $\mathrm{GrC}(\mathrm{aa})-\mathrm{GoC}$ & $320 \pm 230$ & $0.78 \pm 1.1$ & 22000 & $1 \pm 0$ \\
\hline $\mathrm{GrC}(\mathrm{a} a)-\mathrm{PC}$ & $82 \pm 24$ & $0.28 \pm 0.52$ & 20000 & $2.4 \pm 1.1$ \\
\hline $\mathrm{GrC}(p f)-\mathrm{GoC}$ & $910 \pm 360$ & $2.2 \pm 1.6$ & 64000 & $1 \pm 0$ \\
\hline $\mathrm{GrC}(p f)-\mathrm{PC}$ & $1500 \pm 300$ & $5.1 \pm 2.6$ & 140000 & $1 \pm 0$ \\
\hline $\operatorname{GrC}(p f)-B C$ & $740 \pm 130$ & $3.8 \pm 2.1$ & 110000 & $1 \pm 0$ \\
\hline $\operatorname{GrC}(p f)-S C$ & $480 \pm 160$ & $5.1 \pm 3.2$ & 140000 & $1 \pm 0$ \\
\hline BC-PC & $20 \pm 9.9$ & $14 \pm 8$ & 2000 & $1 \pm 0$ \\
\hline SC-PC & $5.4 \pm 2.7$ & $1.8 \pm 1.5$ & 530 & $1 \pm 0$ \\
\hline$B C-B C$ & $14 \pm 6.7$ & $14 \pm 6.6$ & 200000 & $100 \pm 4$ \\
\hline SC-SC & $14 \pm 6.1$ & $14 \pm 5.9$ & 430000 & $100 \pm 4$ \\
\hline GoC-GoC (gap) & $8.4 \pm 3.5$ & $8.4 \pm 3.5$ & 2100 & $3.5 \pm 1.6$ \\
\hline
\end{tabular}


Figure 3 | Network responses to background noise and $\boldsymbol{m f}$ bursts. (a) Power spectra of $G r C$ and

GoC activity are computed with Fast Fourier Transform (FFT) of spike time series (total population spike-counts in $2.5 \mathrm{~ms}$ time-bins). The periodicity of peaks in power spectra reveals synchronous lowfrequency oscillations in the granular layer. The grey curves represent the power spectra when $G o C$ $G o C$ gap junctions were disabled, showing a marked decrease in periodicity. The grey bands correspond to mouse theta-band $(5-10 \mathrm{~Hz})$. (b) The Peri-Stimulus-Time-Histograms (PSTH) of each neuronal population show the effect of the localized $m f$ burst (onset indicated by arrowhead) emerging over background noise. The PSTHs show number of spikes $/ 5 \mathrm{~ms}$ time-bins normalized by the number of cells, averaged over 10 simulations. (c) Example of multiple linear regression of $\mathrm{GrC}$ responses (firing rate) against the number of synaptic spikes from gloms and GoCs, during $40 \mathrm{~ms}$ after stimulus onset. The grey surface is the fitted plane to the points (each point corresponds to a $G r C$ receiving the $m f$ burst on at least 1 dendrite).

a
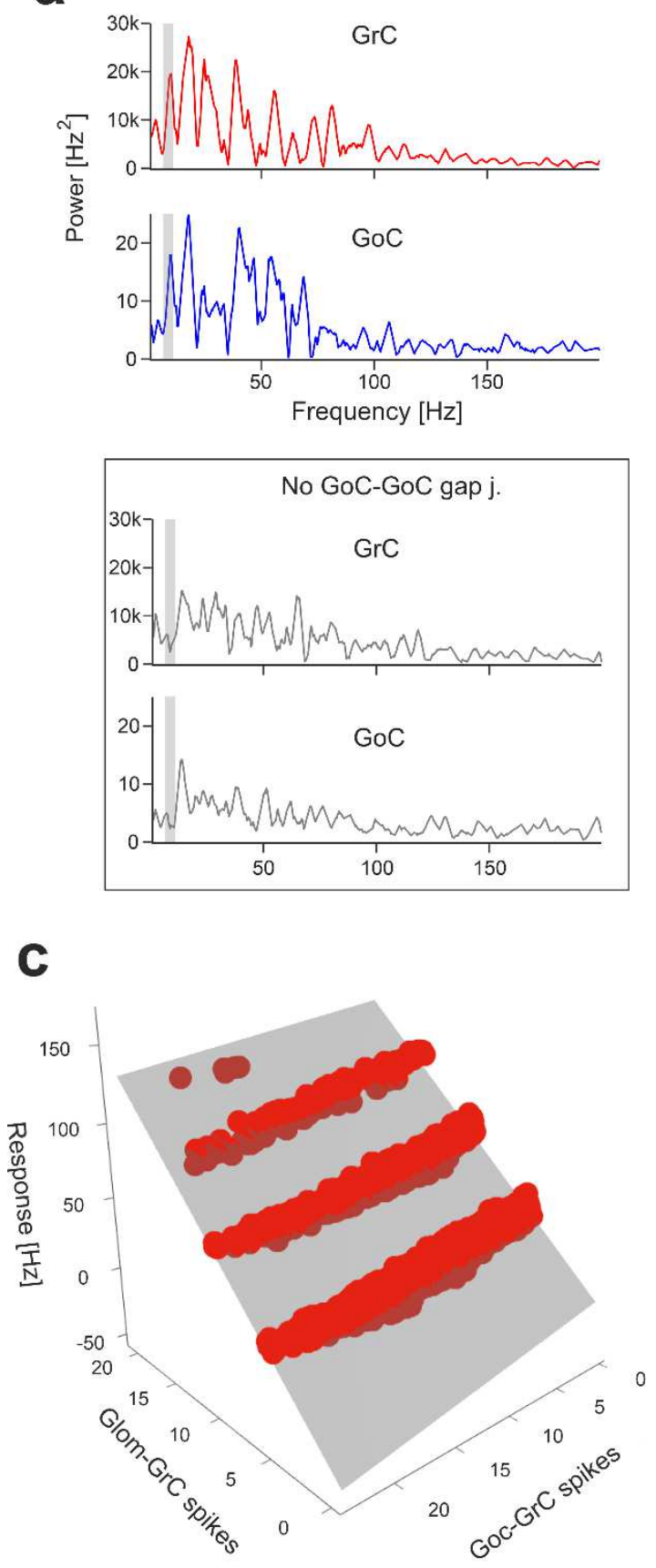

b
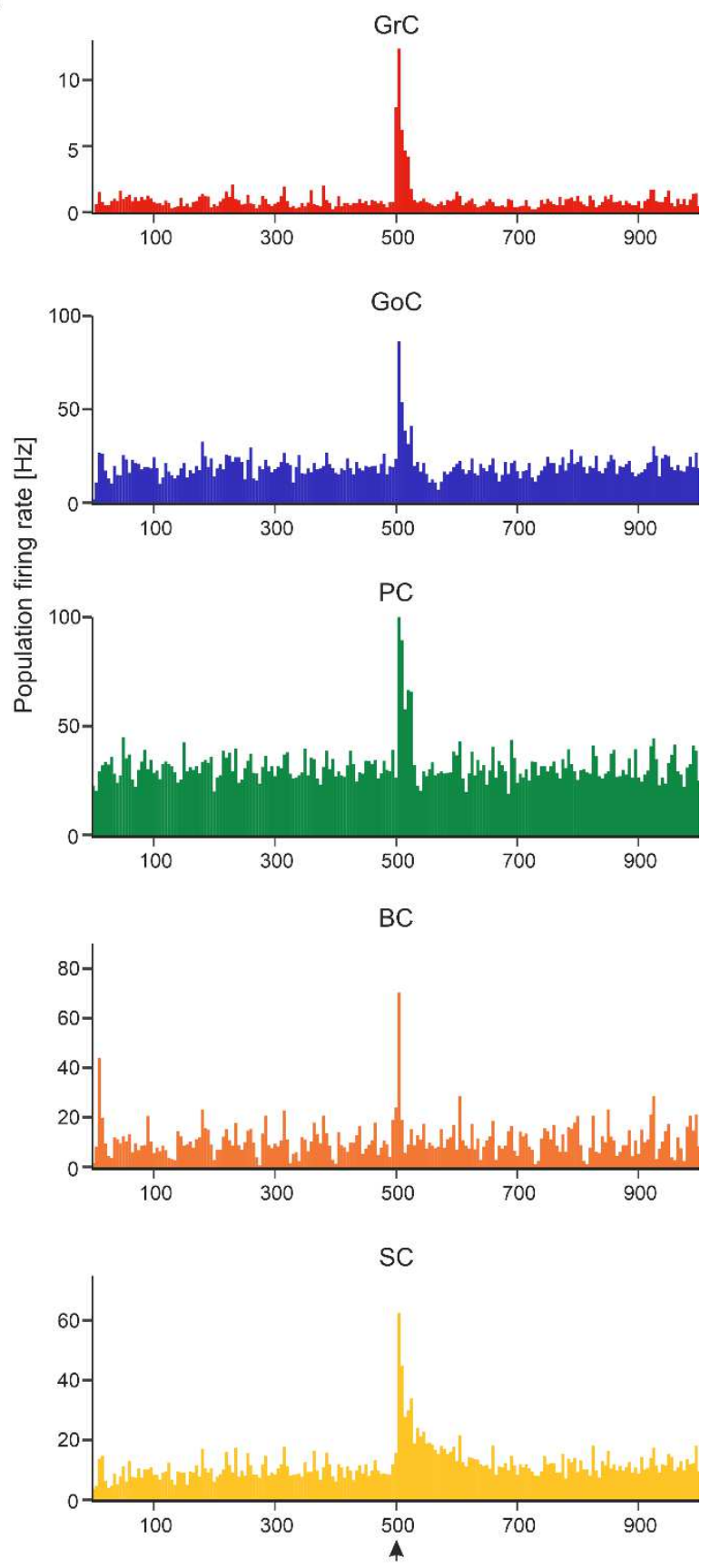

Time $[\mathrm{ms}]$ 
Figure 4 | Granular layer activation. (a) Membrane potential of 4 representative $G r C s$ with 1 to 4 dendrites activated by the $m f$ burst $(20 \mathrm{~ms} @ 200 \mathrm{~Hz}$ over background noise, onset indicated by arrowhead), in control condition and after GABA-A receptors blockade ("GABA-A off"). The burst response of the $G r C$ with 4 active dendrites is enlarged on the right to highlight spike-timing (dashed lines indicate the $m f$ burst spikes). (b) Number of spikes (measured in the $40 \mathrm{~ms}$ from $m f$ burst onset), first spike latency, and dendritic $\left[\mathrm{Ca}^{2+}\right]_{\text {in }}$ (measured in the $500 \mathrm{~ms}$ from $\mathrm{mf}$ burst onset) in subgroups of $G r C s$ with the same number of activated dendrites (mean \pm sd). The graphs compare responses in control and during "GABA-A off". (c) Synapses of a GoC activated by GrCs. Bigger markers correspond to presynaptic GrCs more activated by the $m f$ burst. The GABAergic synapses from other GoCs are on basolateral dendrites, $a a$ synapses are on basolateral dendrites, $p f$ synapses are on apical dendrites. In this example, the $G o C$ receives $30 \%$ of its $a a$ synapses and $6 \%$ of its $p f$ synapses from $G r C s$ with at least 2 active dendrites. Traces on the right show the $G o C$ membrane potential in response to the $m f$ burst (same stimulation as in (a), grey band) in control and during GABA-A receptors and gap junctions switch-off.
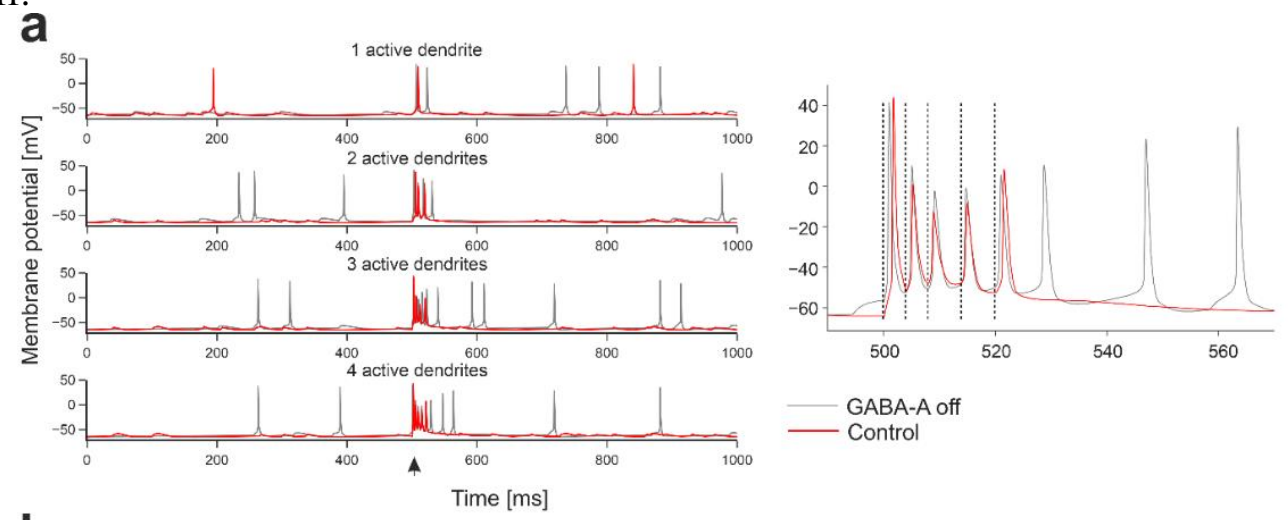

b
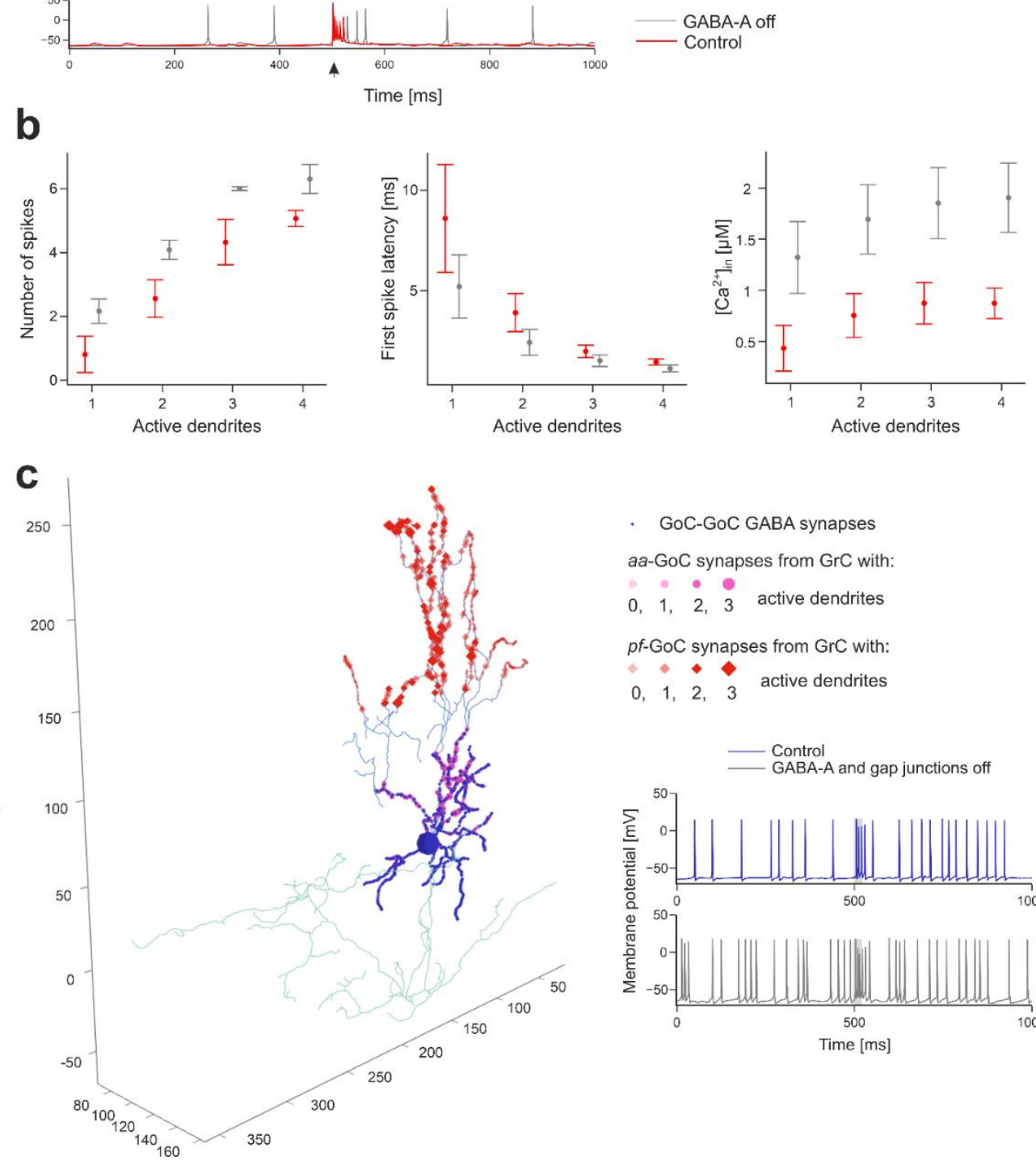
Figure 5 Purkinje cell activation. (a) The $P C$ placed on top of the $G r C$ active cluster and the $P C$ placed at its margin show different synaptic inputs. GABAergic synapses from $S C$ s are on mediumthickness dendrites (those from $B C$ s on $P C$ soma are not shown), $a a$ synapses are located on thin dendrites and $p f$ synapses on thick dendrites. Bigger markers correspond to presynaptic GrCs more activated by the $m f$ burst. In this example, the on-beam $P C$ receives $23 \%$ of its $a a$ synapses and $6 \%$ of its $p f$ synapses from GrCs with at least 2 active dendrites, the off-beam $P C 0 \%$ of its $a a$ synapses and $0.6 \%$ of its $p f$ synapses from $\mathrm{GrCs}$ with at least 2 active dendrites. The corresponding membrane potential traces are shown at the bottom (the $20 \mathrm{~ms} m f$ burst is highlighted by grey band). (b) Analysis of the burst-pause response of PCs to the $m f$ burst (20ms@200Hz over background noise). The burst coefficient (i.e. the shortening of the inter-spike interval due to the $m f$ burst, with respect to baseline) is reported against the number of spikes from $a a$ s and from $p f \mathrm{~s}$ (multivariate regression analysis: $\mathrm{R}^{2}=0.91$ ). The pause coefficient (i.e. the elongation of the inter-spike interval after the $m f$ burst response, with respect to baseline) is reported against either the burst coefficient (NMI=0.79) or the number of spikes from $S C$ s and $B C$ s (NMI=0.66). (c) Synaptic currents recorded from the $P C$ on top of the $G r C$ active cluster (same as in (a)), in voltage-clamp. The traces are the sum of all excitatory (AMPA) and inhibitory (GABA) dendritic currents during the $m f$ burst. They are rectified, normalized and cross-correlated (inset) unveiling a GABA current lag of $2.6 \mathrm{~ms}$ with respect to AMPA current. (d) By stimulating a $m f$ bundle (100ms@50 Hz Poisson stimulation on 24 adjacent $m f \mathrm{~s}$ ), the $P C$ response (modulation with respect to baseline) was quantified by the relative change of Inter-Spike-Interval (ISI), during the stimulus, where 0 corresponds to baseline. The two series of points compare $P C$ response modulation when $S C$ s and $B C$ s were either connected ("control") or disconnected from PCs ("MLI-PC off"). The curves are regression fittings to the points (Kernel Ridge Regression using a radial basis pairwise function, from Python scikit-learn library). The $G r C$ active cluster ("GrC activation") was identified by a threshold on the stimulation-induced activity by using kernel density estimation. 

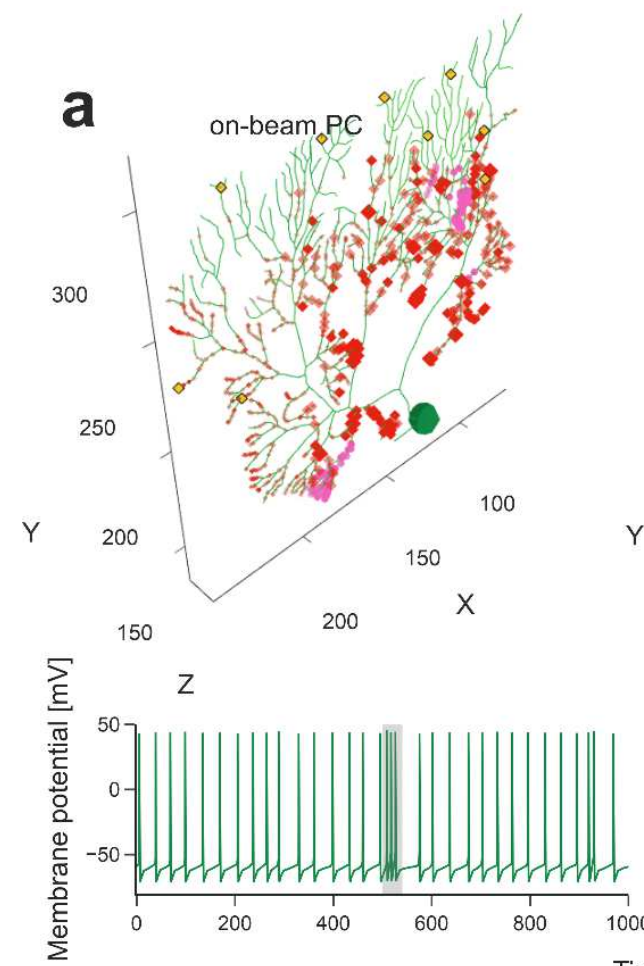

b
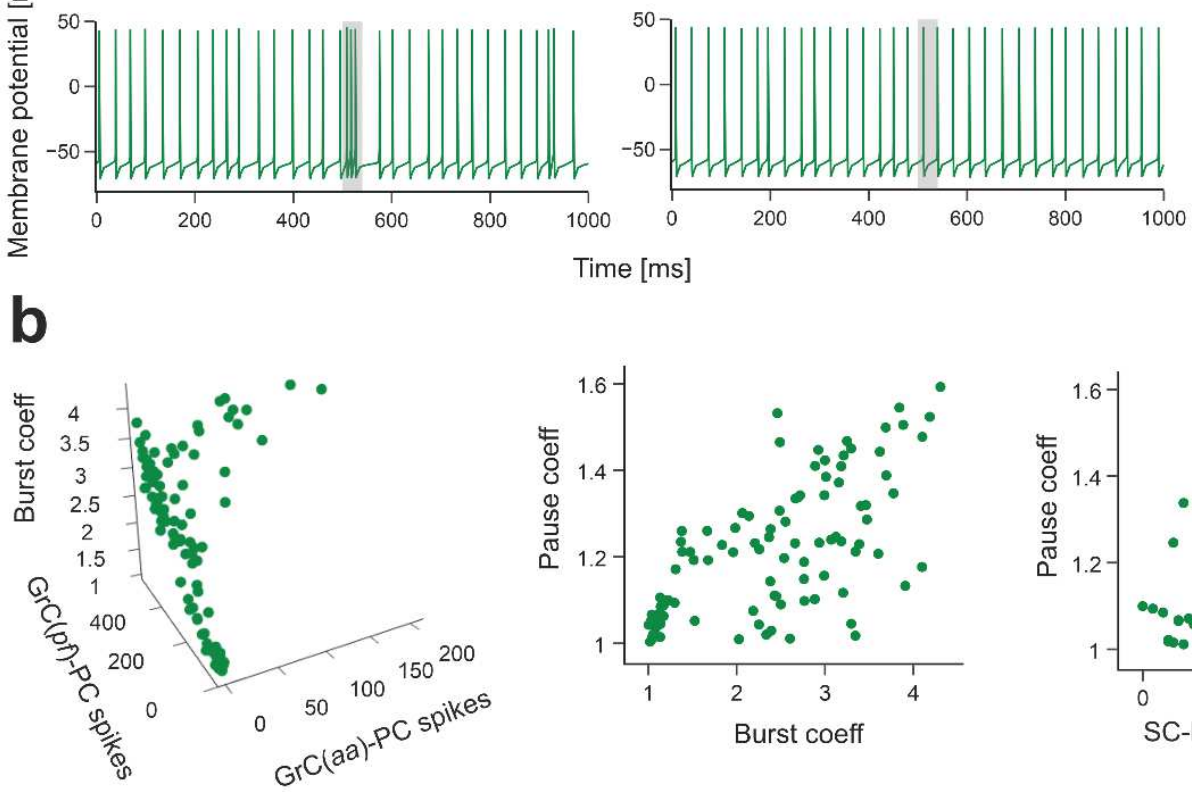

$\diamond \quad$ SC GABA synapses

aa synapses from $\mathrm{GrC}$ with:

0 act. dend.

1 act. dend.

- 2 act. dend.

- 3 act. dend.

pf synapses from $\mathrm{GrC}$ with:

- 0 act. dend.

- 1 act. dend.

- 2 act. dend.

- 3 act. dend.

4 act. dend.
150

250

$\mathrm{X}$

300

200

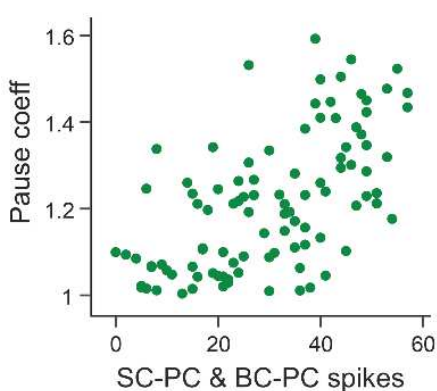

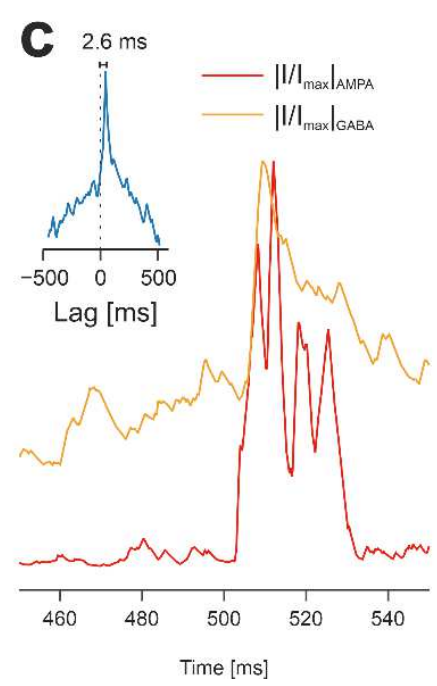

d

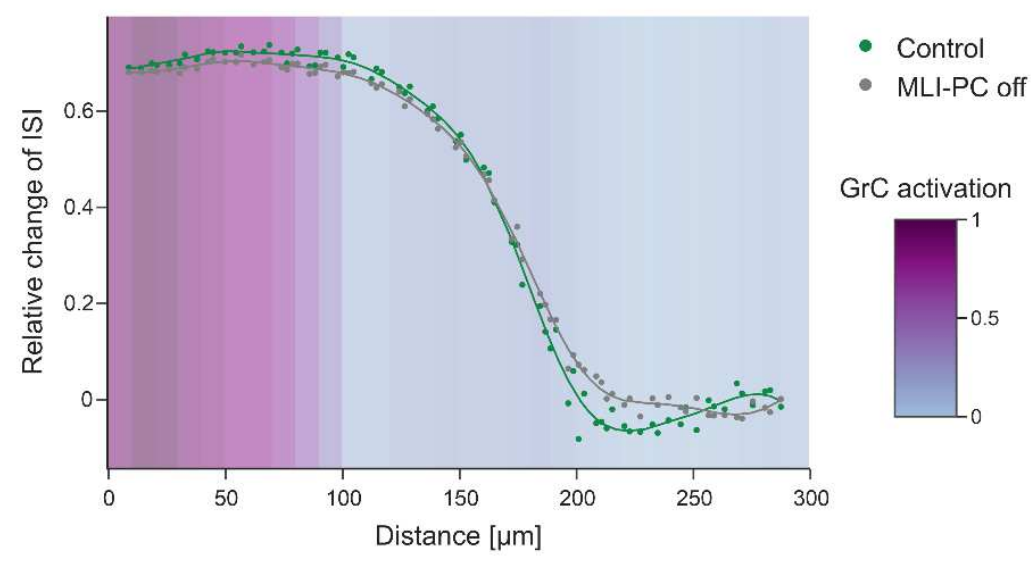


Figure 6 | Activation of a vertical neuronal column in the cerebellar cortex. A whisker air-puff stimulus (the $m f$ burst) is delivered to 4 adjacent $m f$ s, which branch in 4 glom clusters. $G r C$ s respond rapidly with a burst when at least 2 dendrites are activated. A $\mathrm{GrC}$ dense cluster is formed and the signal propagates up through an $a a$ bundle and transversally along a $p f$ beam. GoCs receive the signal both on basolateral and apical dendrites. $P C$ s vertically on top of the active cluster are invested by $a a$ and $p f$ synaptic inputs. On-beam $S C$ s and $B C$ s receive signals through $p f$ synapses; $S C$ axons inhibit mainly on-beam $P C$ s, while $B C$ axons inhibit mainly off-beam $P C$ s. The membrane potential traces $(m f$ burst starts at $500 \mathrm{~ms}$ ) are shown for each neuronal population. Traces in the three columns correspond to three different release probabilities at the $m f-G r C$ synapses: $\mathrm{u}=0.1, \mathrm{u}=0.43$ (control condition used in the rest of the paper), $\mathrm{u}=0.9$. The lower and higher $\mathrm{u}$-values are typical of long-term synaptic depression and potentiation in the granular layer.

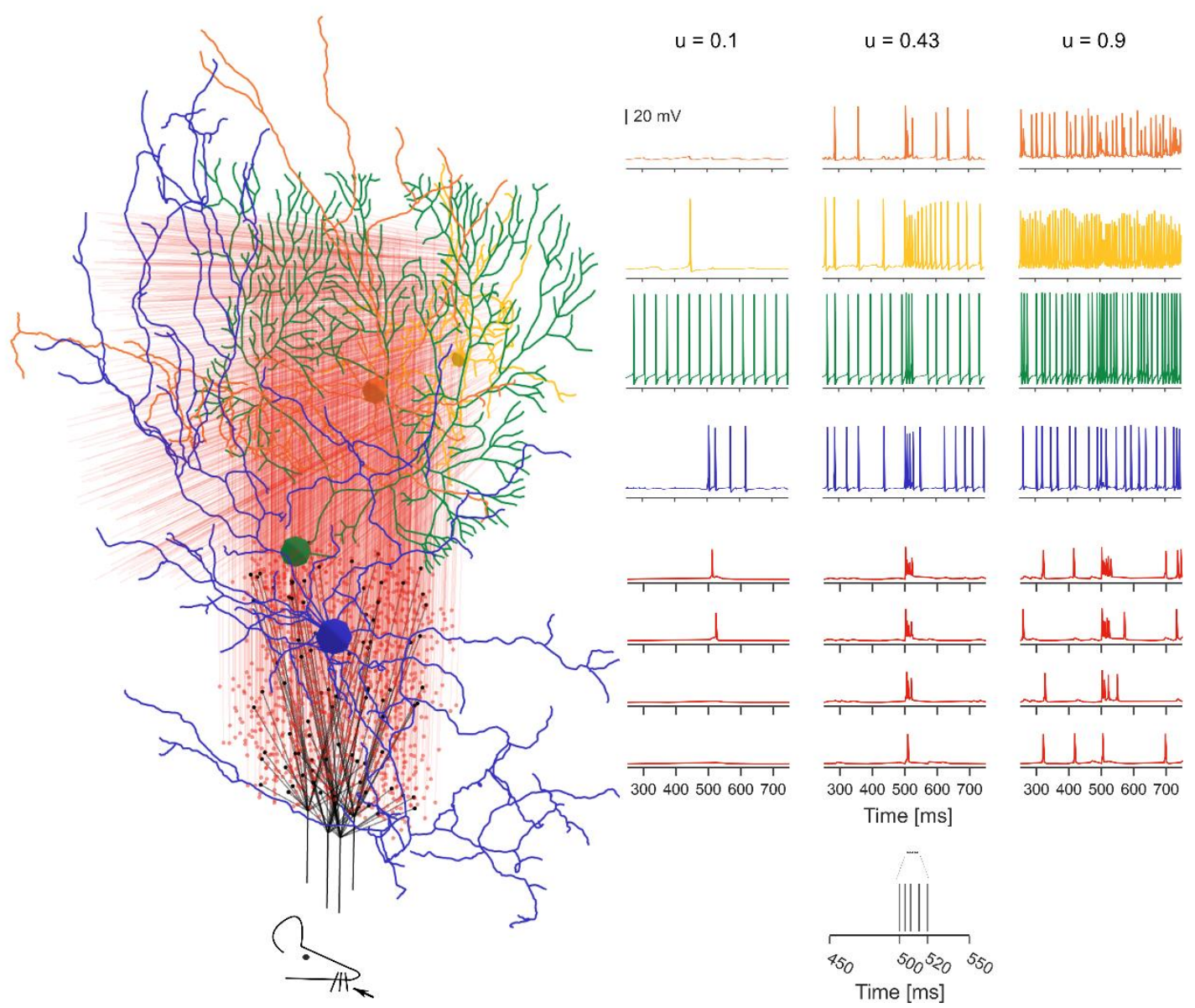


1043 Supplementary figures and videos can be visualized as interactive .html files at https://dbbs1044 lab.github.io/deschepper-etal-2021/, adding e.g. "video" + number + ".html" e.g.: "videoS2.html", on 1045 this URL.

Figure S1 | Placement metrics. Cell placement is assessed using various metrics for each population, including (a) Nearest Neighbor distance, (b) Pairwise Distance, (c) Radial Distribution Function. These metrics show realistic cell positioning.

1050

a Nearest neighbour
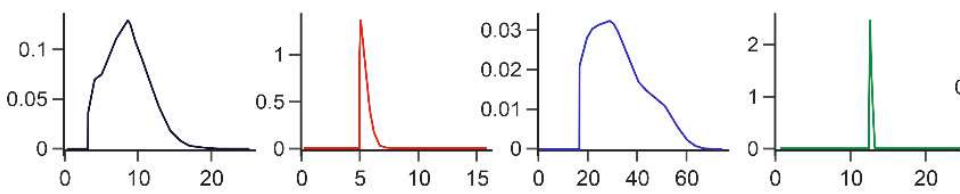

Distance $[\mu \mathrm{m}]$

b Pairwise distance
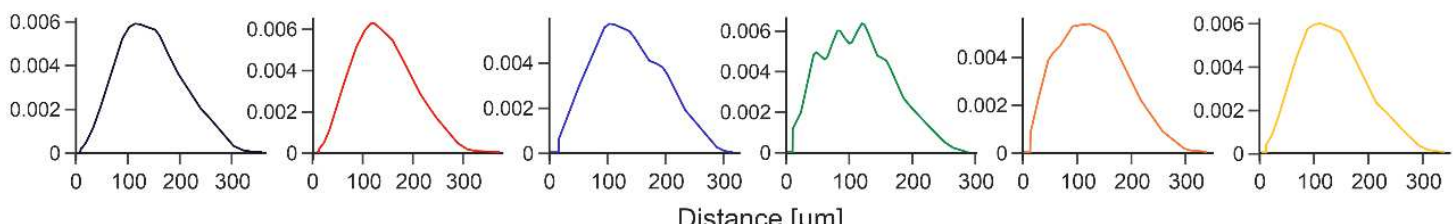

Distance $[\mu \mathrm{m}]$

C Radial distribution function

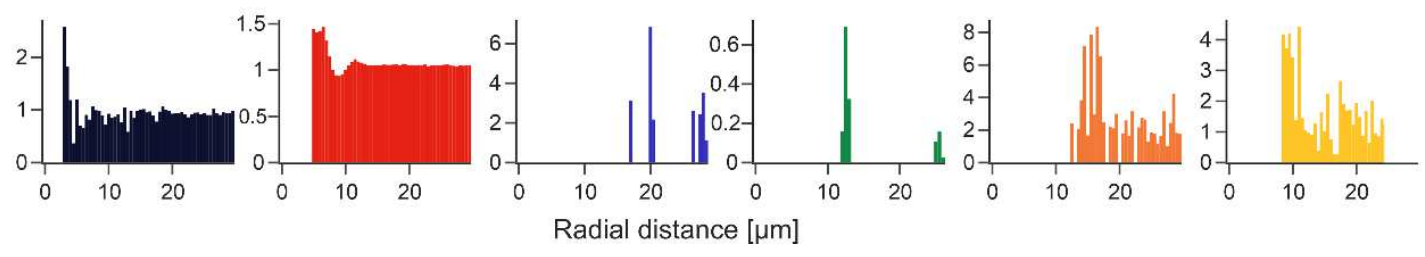


Figure S2 | Connecting SC-PC by voxel intersection. A mesh of adjacent voxels is used to enwrap the axon of a stellate cell (50 cubes with $4.6 \mu \mathrm{m}$ side) and the dendritic tree of a $P C$ (50 cubes with 26 $\mu \mathrm{m}$ side). The intersecting voxels are in red. The synapses are located on compartments within the intersecting voxels.

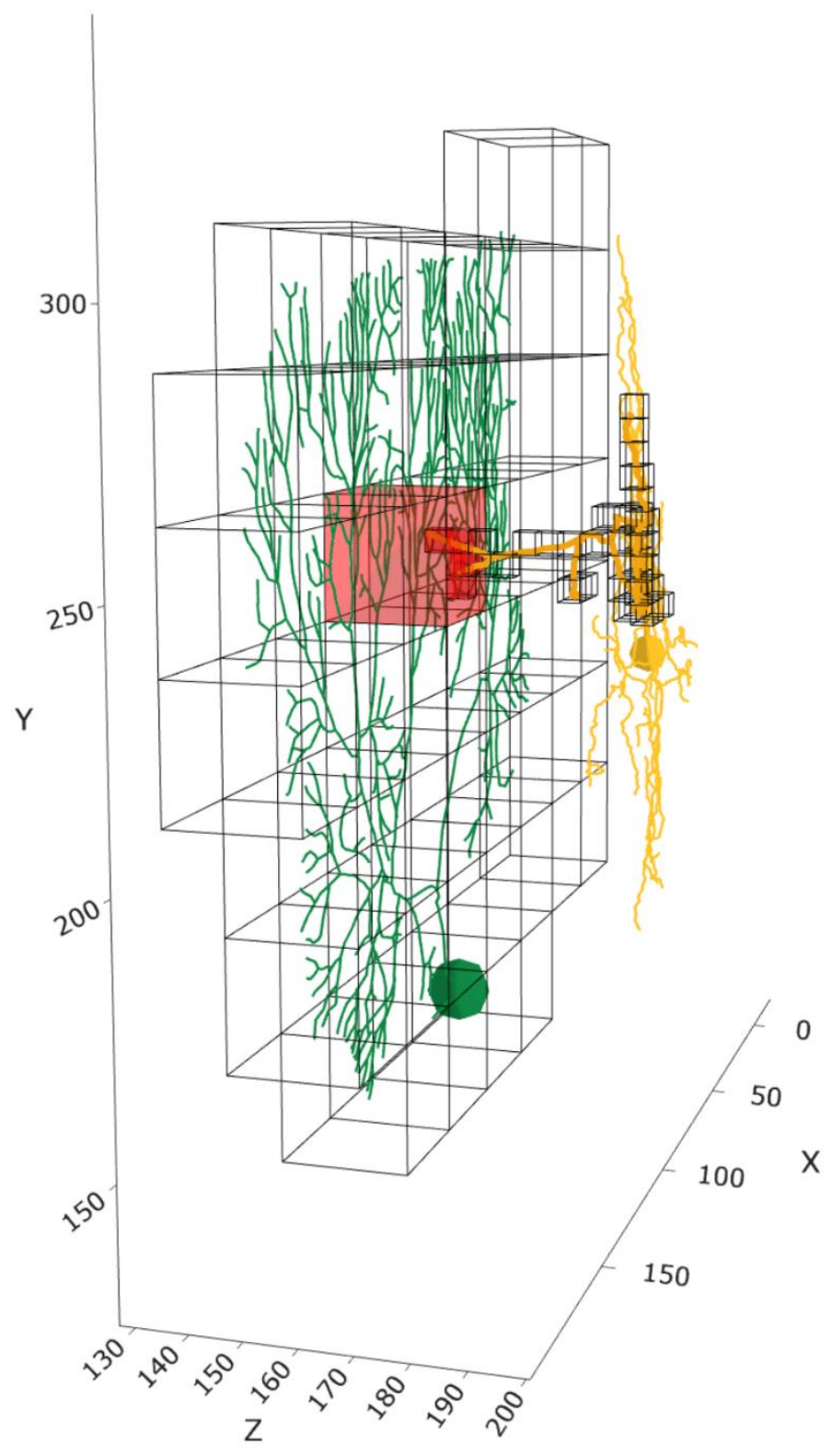


Figure S3 $\mid$ MLI responses to $\boldsymbol{m f}$ burst. (a) Multiple linear regression of $S C$ s and $B C$ s in responses to the $m f$ burst against the number of synaptic spikes from $p f \mathrm{~s}$ and from other $S C \mathrm{~s}$ or $B C$ s. (b) One $S C$ and one $B C$ crossed by an active $p f$ beam are represented in $3 \mathrm{D}$. The GABAergic synapses from other $S C \mathrm{~s}$ or $B C \mathrm{~s}$ are also indicated. Bigger markers correspond to presynaptic $\mathrm{GrCs}$ more activated by the $m f$ burst. In this example, the $S C$ receives $8 \%$ and the $B C 7.5 \%$ of their $p f$ synapses from $G r C s$ with at least 2 active dendrites.

a
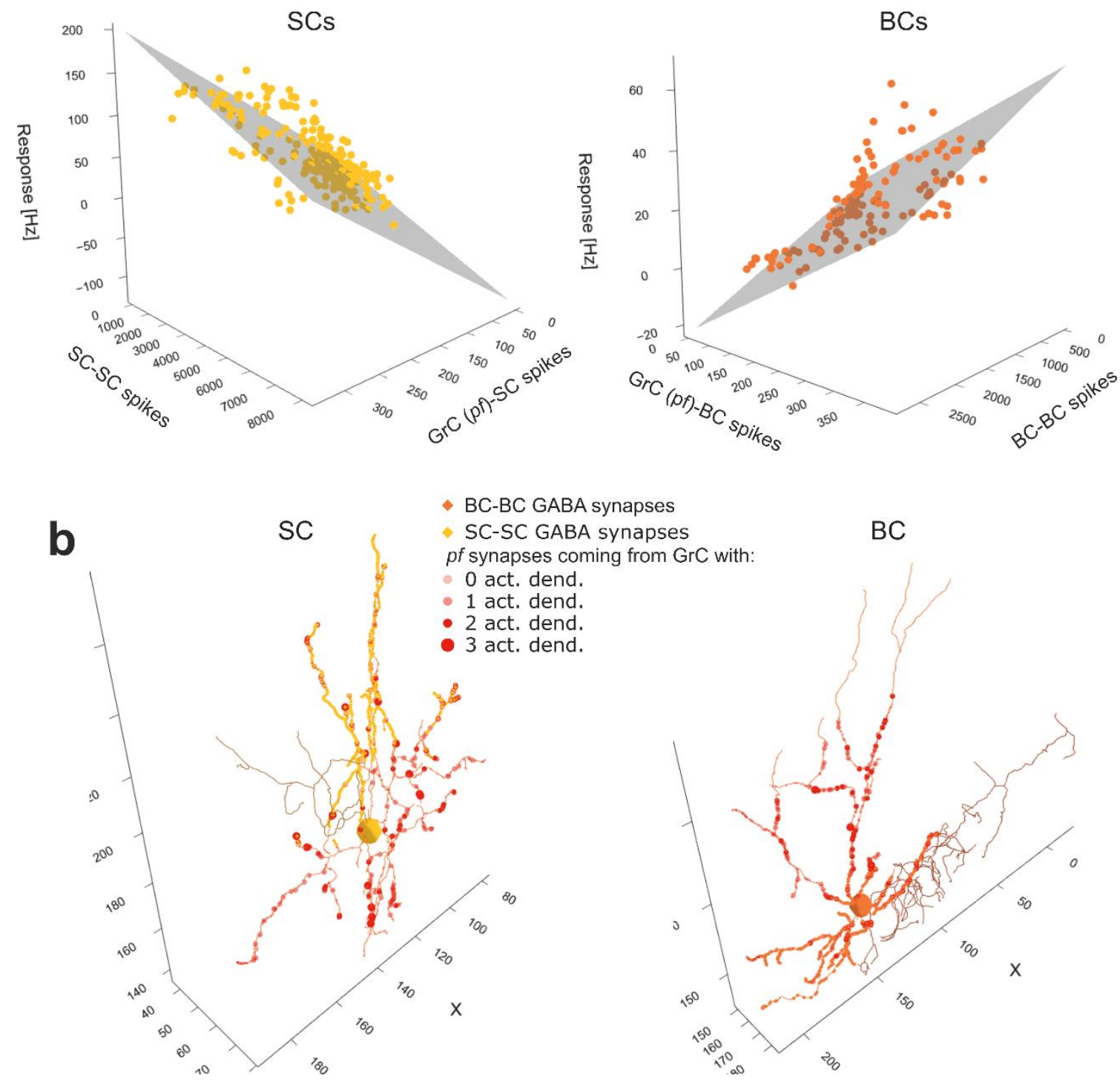

1067 
1069

1070

1071

1072

1073

1074

1075

1076

Figure S4 | Input-output dependency of model neurons. Multiple linear regression between neuronal responses (firing rates) to the $m f$ burst and the number of incoming spikes from the presynaptic neurons (during $40 \mathrm{~ms}$ from the $m f$ burst onset), averaged over 10 simulations. $\mathrm{R}^{2}$ and direction coefficients are reported.

\begin{tabular}{|c|c|c|c|}
\hline & $\mathrm{R}^{2}$ & & Coefficient \\
\hline \multirow{2}{*}{ GrC } & \multirow{2}{*}{0.91} & Glom & 6 \\
\hline & & GoC & -0.55 \\
\hline \multirow{4}{*}{ GoC } & \multirow{4}{*}{0.81} & Glom & 0.14 \\
\hline & & $\mathrm{GrC}(\mathrm{aa})$ & 0.15 \\
\hline & & $\mathrm{GrC}(\mathrm{pf})$ & 0.1 \\
\hline & & GoC & -0.006 \\
\hline \multirow{4}{*}{$P C$} & \multirow{4}{*}{0.95} & $\mathrm{GrC} \mathrm{(aa)}$ & 0.057 \\
\hline & & $\mathrm{GrC}(\mathrm{pf})$ & 0.091 \\
\hline & & SC & 0.074 \\
\hline & & $\mathrm{BC}$ & -0.2 \\
\hline \multirow{2}{*}{ SC } & \multirow{2}{*}{0.79} & $\mathrm{GrC}(\mathrm{pf})$ & 0.54 \\
\hline & & SC & -0.018 \\
\hline \multirow{2}{*}{$B C$} & \multirow{2}{*}{0.72} & $\mathrm{GrC}(\mathrm{pf})$ & 0.11 \\
\hline & & $\mathrm{BC}$ & -0.017 \\
\hline
\end{tabular}


Movie S1. Impulsive response of the cerebellar network. The movie shows the activation of the cerebellar network receiving (at movie time $=12 \mathrm{~s}$, indicated by a green light) the $m f$ burst (20ms@200 $\mathrm{Hz}$ on a bundle of adjacent $4 \mathrm{mfs}$ ) to emulate whisker/facial sensory stimulation in vivo, superimposed on diffused background noise ${ }^{20}$. Note the sparse activity in $\mathrm{GrCs}$ that suddenly aggregates into dense clusters activating the overlaying $P C$ s and MLIs. This pattern gives rise to a vertical column of activity in the cerebellar cortex (see also Fig.6). It should be noted that, compared to ${ }^{14}$, the granular layer occasionally activates clusters of GrCs under background bombardment due to the $\mathrm{mf}$-glom ramifications and clustering that privileges the areas where gloms originating from the same $m f \mathrm{~s}$ overlap. Nonetheless, this activity is rarely strong enough to cause a visible activation of the overlaying $P C$ s and $M L I$ s.

Movie S2. $\mathrm{GrC}$ response. The electrical activity of a $\mathrm{GrC}$ embedded into the cerebellar network (same cell as in Fig. 4a with "4 active dendrites") is animated along with dynamic changes in molecular and synaptic variables. The movie shows $100 \mathrm{~ms}$ of simulation, with the $m f$ burst $(20 \mathrm{~ms} @ 200 \mathrm{~Hz}$ on a bundle of adjacent $4 \mathrm{mfs}$ superimposed on diffused background noise) starting at movie time $=5 \mathrm{~s}$ (indicated by a green light). Each variable is reported on the $\operatorname{GrC}$ morphology (4 dendritic compartments, soma, axon hillock, ascending axon, parallel fiber) using a colour scale. $\left(\mathbf{V}_{\mathbf{m}}\right)$ membrane potential; (Ca) intracellular calcium concentration; (AMPA), (NMDA) and (GABA), synaptic currents recorded in voltage-clamp.

Movie S3. GoC response. The electrical activity of a $G o C$ embedded into the cerebellar network (same cell as in Fig. 4c) is animated along with dynamic changes in molecular and synaptic variables. The movie shows $100 \mathrm{~ms}$ of simulation, with the $m f$ burst $(20 \mathrm{~ms} @ 200 \mathrm{~Hz}$ on a bundle of adjacent $4 \mathrm{mfs}$ superimposed on diffused background noise) starting at movie time $=0 \mathrm{~s}$ (indicated by a green light).Each variable is reported on the $G o C$ morphology (using a colour scale) and shown at the bottom with traces (using a moving time window) referring to specific neuron compartments: the soma (teal), an apical dendrite (pink) and a basolateral dendrite (gold). ( $\left.\mathbf{V}_{\mathbf{m}}\right)$ membrane potential traces in the soma and apical dendrite. (Ca) $\left[\mathrm{Ca}^{2+}\right]_{\text {in }}$ in the basolateral and apical dendrite. (AMPA) synaptic current in the basolateral and apical dendrite (synapses from $m f s$, $a a$ s, and $p f s$ ). (NMDA) synaptic current in the basolateral dendrite (synapses from $m f \mathrm{~s}$ and $a a \mathrm{~s}$ ). (GABA) synaptic current in the basolateral dendrite (synapses from $\mathrm{GoCs}$ ). 


\section{Supplementary Files}

This is a list of supplementary files associated with this preprint. Click to download.

- movieS2networkActivity.mp4

- movieS3GrC.mp4

- movieS4GoC.mp4 